\title{
Basic Quantum Field Model of the Self-Organization of Microtubules in Eukaryotic Cells
}

\author{
Paul Levi \\ Department for Informatics, Faculty for Informatics, Electrical Engineering and Information Technology, University Stuttgart, Institute for \\ Parallel and Distributed Systems, Stuttgart, Germany
}

Email address:

paul.levi@ipvs.uni-stuttgart.de

\section{To cite this article:}

Paul Levi. Basic Quantum Field Model of the Self-Organization of Microtubules in Eukaryotic Cells. European Journal of Biophysics. Vol. 8, No. 2, 2020, pp. 60-75. doi: 10.11648/j.ejb.20200802.17

Received: August 31, 2020; Accepted: October 13, 2020; Published: November 23, 2020

\begin{abstract}
The background of this contribution is the ongoing extended debate over quantum effects in the biology since decades. Typical examples of the quantum biology are photosynthesis, enzymatic activities, bird navigation and especially the coherent movement of microtubules. The reason for all these effects is dominantly the quantum coherence of waves. The objective of this contribution is the quantum description of the instability dynamics of microtubules at their assembly and disassembly phases during the interphase. The corresponding theoretical investigations of this article confirm the existence of quantum coherence of microtubules. Experimental results assert such vibrations of microtubules by the observation of $\gamma$-waves in the human brain generated by bundles of microtubules. Tubulin subunits and the accessory proteins are of nano size; therefore, they are modeled as field quanta in the framework of non-relativistic quantum field theory. This approach describes the dynamics of these quantum particles and their interactions, by accentuation of their different performances as coherent or incoherent waves. The achieved results strongly depend from the preconditions: whether the fluctuating forces are turned on or turned off. With the inset of fluctuations, the quantum coherence is destroyed, and only incoherent particle solutions are obeyed. Without the impact of fluctuations wave solutions dominate. Another type of wave solution are coherent wave packets which are counter-running, where their superposition can extinct or enhance them. This kind of interfering coherent solutions is applied on the polymerization of protofilaments. The calculations of this contribution demonstrate that the quantum coherence can be only observed when fluctuations are excluded. The conclusion is that that dedicated biological processes must be able to suppress the destroying influences of the local environment. In contrary to technical-based experiments, where coherence is only obtained when the fluctuations are deliberately excluded (e.g. quantum computer). Therefore, the answer why the processes of quantum biology can generate quantum coherence at least in case of microtubules is actually not answered. Kinesins in combination with microtubules are fundamental for cellular functions and morphogenesis. Recent genetic experiments uncovered their role for tumor suppression and developmental patterning. However, these findings which open exciting new areas of kinesin research are not included in this contribution, because the description of the kinesinmicrotubule system is to comprehensive for one article.
\end{abstract}

Keywords: Microtubules, Dynamic Instability, Quantum Coherence, Self-Organization

\section{Introduction}

The remarkable system of microtubules, actin filaments and intermediate filaments is called cytoskeleton [1, 2], where this contribution concentrates to microtubules, Possible disorders of them and medical implications are in the actual focus of research activities [3].

They are dynamic and adaptable with respect to their assembly and disassembly and they direct the intracellular transport. However, the microtubules are without the large set of asseccory proteins which are essential for the regulated assembly or disassembly at particular locations (selforganization). Thus, the role of these proteins is comparable to the set of proteins that bind to an enhancer DNA sequence and to the plenty of transcriptional repressors [4].

Typical already approved quantum processes that occur in biological processes are the photosynthesis, enzymatic activities, sense of smell, bird navigation (compass in bird 
eyes) and finally the coherent vibration of microtubules. All aforementioned processes belong to quantum biology [5], where the last-mentioned effect was strongly advocated by Penrose [6], who was harshly criticized by opponents of quantum biology, because they consider the brain is too warm, wet and noisy for selected quantum processes. Thus, the established quantum physics exclude the quantum coherence under such conditions. However, for instance the discovery of quantum vibrations in microtubules contradict this statement. Because there exists experimental evidence that bundles of microtubules (amplification effect) generate oscillations that are generated by quantum coherence and are measurable as EEG waves ( $\gamma$-waves) $[7,8]$.

Thus, it is astonishing that the aforementioned quantum processes are confirmed, although they are all mainly initiated by the effect of quantum coherence. The corresponding conclusion is that the effect of decoherence does not occur at special cases in cells. Although, experimental results and simulations, e.g. [9] demonstrate the quantum coherence e.g. of microtubules, although the reason of this effect is not well established. So, three oppositional explanations exist. The first statement claims that consciousness is closely connected with quantum coherence of microtubules (Penrose). The second assertion asserts that coherent quantum waves can superpose and reinforce themselves to. wave crest or even to "quantum tsunamis" which suppress the destroying influence of noise, at least for a short duration. The third clarification explicate the possibility that a kind of a sheltering wall is established by nature which prevent the destroying forces of the local environment, as for instance it occurs in case of superconductivity. Which proposal is valid is currently not decided.

Independent of the proposals about the origin of quantum coherence it seems obvious that coherent waves envelope several free molecules (e.g. tubulin subunits) at once and compose them together (assembly) more quickly than by a regular particle by particle assembly (e.g. elongation of $1 \mu \mathrm{m} / \mathrm{min}$ ). Inversely, the breakdown of a coherent wave can accelerate the disassembly of a microtubule (e.g. standard value $7 \mu \mathrm{m} / \mathrm{min}$ ). Both effects can be applied to make corresponding measurements or perform suitable simulations.

Two previous publications considered the one-arm (motion of a myosin head on actin filaments [10] and the bipedal ("legover-leg") walk of dynein along microtubules [11]. In both cases the applied fundamental methods are based on nonrelativistic Quantum Field Theory (QFT) [12]. It is remarkable that the solutions of myosin and dynein despite the different mechanisms of movement, include also coherent waves results (quantum coherence). Thus, the wave like results of the motion of dynein supplement the quantum coherence of microtubules which are elaborated in this contribution.

The focus of this paper directs to dominant aspects of the motility of eukaryotic cells originating from microtubules. Thus, the essential topic of this contribution is the quantum mechanical description of the self-organizing processes caused by the dynamics of the polymerization (assembly) and depolymerization (disassembly) of microtubules during the interphase $\left(G_{1}, S\right.$ and $G_{2}$ phases $)$ of a cell cycle [13]. The quantum dynamics of processes that are also reported are the polymerization of tubulin subunits, the assembly of protofilaments together with their nucleation at the MTOC (microtubule-organizing center) and the motion of free subunits to the locations at nascenting microtubules.

Microtubules are often considered in connection with the essential motor protein kinesin. Cytosolic kinesin moves along microtubules and transport heavy molecules (e.g. organelles, vesicles) from the minus end of a microtubule to the plus end (anterograde transport) through a crowded cytosol. The cytoplasmatic dynein carry for instance vesicles toward to the minus end of a microtubule (retrograde transport).

Mitotic (spindle) kinesin transports for instance chromosomes along microtubules, assembles spindles and attach chromosomes to spindles. Thus, both types of kinesin are an integral operative during mitosis.

Since about two decades exciting and extended experimental results and profound theoretical investigations of different kinesin-microtubules systems have been published [14-16]. These endeavors concern, for example the kind of movements (mechanochemical cycle) of 14 different family members of kinesin, where each member of the kinesin family transports a specific cargo. Astonishing details of cargo transports and the unexpected role of kinesin are observed in the regulation of physiological processes, tumor suppression and developmental patterning [17].

This contribution mainly concentrates to the assembly respectively the disassembly of microtubule in the interphase. The consideration of the relevant transport features of kinesin along microtubules are not considered, because the integration of the great amount of their complex transport details is beyond the scope of this paper. It requests a new separate treatment.

The fascinating effect of the self-organization of micro tubules is in biological view comparable with the swarm behavior of bees or ants. These insects are small and operate well organized to construct a huge "building" without using a predefined plan, where the established nests with separate cells react (backlash) on them.

The basic mathematical principles underlying the selforganized molecular processes of microtubules, is formally characterized by the synergetic approach [18]. However, this approach is only descriptively included in this contribution, because its emphasis lies in quantum-based descriptions of special cytoskeletal processes and not in the strong mathematical description of synergetics. So, for instance, the introduction of control and order parameters is typical for the synergetic approach. For example, ferromagnetic magnetic material becomes magnetic (order parameter) when the elementary magnets are aligned if $\mathrm{T}<\mathrm{T}_{\text {cr }}$, where $\mathrm{T}_{\text {cr }}$ is the critical temperature (control parameter). Consequently, they are arranged in aligned order. Analog ordering processes occur when water freezes to ice.

Thus far, the role of accessory proteins is not integrated in the classical concept of synergetics, which was developed for example by reference to the Brusselator or the Belusov- 
Zhabotinski reaction [18]. In the context of microtubules these proteins are called MAPs (Microtubule associated proteins) with respect to their importance. MAPs regulate the assembly and disassembly of microtubules, organize them in bundles, cross-link them with membranes, and direct the intracellular transports [1].

The concept of a biological self-organization represents an extension of the original draft of synergetics, where the supply rate (e.g. per second) of the set of MAPs which are focused to a particular task e.g. to the assembly of microtubules is considered as an order parameter [19], or terser expressed as an order parameter field [20]. Since, above the critical concentration of free tubulin subunits (control parameter) all subunits in polymerized microtubules point in the same direction, and all protofilaments of a microtubule are aligned in parallel. Below this critical concentration of subunits, the microtubes depolymerize (disassemble).

Another important aspect of synergetics which also can be applied to quantum biology is the description of the assembly processes respectively disassembly processes as nonequilibrium phase transitions far from the thermal equilibrium. These kinds of transitions occur since cells are open systems.

\section{Molecules and Processes}

The building block of a microtubule is the tubulin subunit, a heterodimer formed from a pair of the monomers $\alpha$-and $\beta$ tubulin, which are tightly connected (non-covalent bond), where the $\beta$-tubulin builds the "top" (surface) of the subunit. The width of the tubulin subunit is $4 \mathrm{~nm}$, whereas its height amounts $8 \mathrm{~nm}$; thus, the heterodimeric subunits repeat every $8 \mathrm{~nm}$ and gives each protofilament a reiterated, elongated structure. The assembled protofilament has two different ends: $\alpha$-tubulin end ( - -) end) and a $\beta$-tubulin "top" $(+)$ end. Thus, the protofilament gets a distinct structural polarity. The tubulin subunit originating by the polymerization of the $\alpha$ and $\beta$-tubulin exists in two conformations. The $\alpha$-tubulin binds the GTP molecule irreversibly and does not hydrolyze, because it is trapped at the interface between the $\alpha$-and $\beta$ tubulin. The $\beta$-tubulin binds GTP reversibly and hydrolyzes it to GDP. This binding site is called the exchangeable site, because GTP can be exchanged by GDP. A microtubule is a polymer, arranged in a cylindrical spiral that consist in the most cases of 13 elongated protofilaments that associate laterally into a hallow spiral. The length of such a tube varies from a fraction of one $\mu \mathrm{m}$ to hundreds of $\mu \mathrm{m}$. The outer diameter amounts about to $25 \mathrm{~nm}$ and the inner diameter is roughly $14 \mathrm{~nm}$ [13].

In the interphase the assembly of a microtubule mesh, with long fibers that fill the whole cytosol, is created. All these tubulin fibers radiate from a central point, the MTOC which lies near the nucleus. This center nucleates the microtubules and it is supported by MAPs which organize the assembly and disassembly in eukaryotic cells, and regulate the number, the forms and the stability of them. Thus, MTOC arranges a wide variety of assembled microtubules with high order; in turn, this center represents an ordered lattice.

Every accessory protein represents signal proteins (signaling pathway) that deliver information (message) to receptor molecules, which convert these signals in cytoskeletal actions. Typical MAPS are the $\gamma$-tubulin (supports the emanation of a microtubule from the surface of the MTOC), the MAP-2 cross-links different microtubules and $\tau$ bundles parallelly various microtubules. MAPS are generally only few $\mathrm{nm}$ in size, the tubulin subunit and the free $\alpha$ and $\beta$-tubulin molecules are from the same small nano size, therefore their movement within the cytosol is described by quantum equations of motions, which excludes the transport of cargo by dynein or kinesin. Usually, molecular motions are described by a classical diffusion. The extension of this standard approach to quantum diffusion or even to the process of multiple scattering (Green's function) is apparently [21]. However, both approaches do not include the process of quantum coherence which is the focal point of this contribution, without considering cargo transports.

The continuous flux of MAPs generates a set of assignments which stipulate special actions. The dynamical equilibrium of a cell is established by this flow which is comparable to a concerted plan (e.g. a musical score for an orchestra), which bring microtubules structures under the control of intracellular signals that trigger the essential transformations during each cell cycle. The continuity of the different, dynamical coherent supply rates of MAPs, concerning different processes (e.g. assembly MAPs), represent order parameters, because there orchestrated acting enables a eukaryotic cell to generate and maintain highly-organized structures that are flexible. The creation of highly organized systems is characteristically for non-equilibrium transitions [20].

The synergetic principle differentiates between one or more control parameters. One control parameter is the critical concentration $\mathrm{C}_{\mathrm{cr}}$ ( $\mathrm{su}$ ) of the free subunits, because below this concentration the disassembly (depolymerization) of microtubules occur and above $\mathrm{C}_{\mathrm{cr}}$ ( $\mathrm{su}$ ) their assembly (polymerization) take place. Thus, the system performance distinctly behaves below and above this particular concentration. The definition of the critical concentration is given by the loss-rate constant $r_{\text {loss }}$ of subunits divided by the addition-rate constant $r_{\text {add }}$ of subunits; that is $\mathrm{C}_{\mathrm{cr}}(\mathrm{su})=\mathrm{r}_{\text {loss }}$ (su) $/ r_{\text {add }}$ (su) [1]. This concentration equilibrium is reached when the disassembly of the polymer back to monomers precisely balance the assembly of monomers to the polymer (association rate $=$ dissociation rate). With respect to free subunits the value of $\mathrm{C}_{\mathrm{cr}}(\mathrm{su})$ defines the concentration of free subunits left in cytosol at this point.

Thus far, the critical fact of nucleotide hydrolysis has not been considered. A free tubulin subunit is usually not hydrolyzed; thus, it still contains the GTP. Such soluble subunits are in T-forms. A non-soluble heterodimer carries the GDP (hydrolyzation of GTP) and is called D-form. For example, during the growth of a microtubule that is not yet nucleated with the minus end at the MTOC, T-forms are added at the plus end of the tubulin polymer and D-forms leave it at the minus end. Hence, $\mathrm{C}_{\mathrm{cr}}$ ( $\mathrm{su}$ ) defines an unstable, dynamical equilibrium because a released $\mathrm{D}$-form is replenished by a free $\mathrm{T}$-form. Near $\mathrm{C}_{\mathrm{cr}}$ ( $\mathrm{su}$ ) microtubules 
alternately grow or shrink, because at a subunit's concentration below $\mathrm{C}_{\mathbf{c r}}$ (su) no polymerization occurs. At a dimers concentration above $\mathrm{C}_{\mathrm{cr}}$ ( $\mathrm{su}$ ) the tubulin subunits polymerize (assemble) into microtubules, where the added $\mathrm{T}$ forms transform into D-forms.

However, the critical concentration of subunits represents a general threshold which is extended by two critical concentrations for $\mathrm{T}$-forms and $\mathrm{D}$-forms. The critical concentration $\left\langle n_{\mathrm{T}}\right\rangle_{\mathrm{cr}}=\left\langle n_{\mathrm{T}}\right\rangle_{\text {loss }} /\left\langle n_{\mathrm{T}}\right\rangle_{\text {add }}$ of T-form polymers is equal to the constant ratio (equality of shrinking and elongation) of the loss rate divided through the addition rate of T-form subunits. Below $\left\langle n_{\mathrm{T}}\right\rangle_{\text {cr }}$ the polymer shrinks (loss of T-forms), above $\left\langle n_{\mathrm{T}}\right\rangle_{\text {cr }}$ it elongates (addition of T-forms). In analogy, the critical concentration of a D-form microtubule reads $\left\langle n_{\mathrm{D}}\right\rangle_{\text {cr }}=\left\langle n_{\mathrm{D}}\right\rangle_{\text {loss }} /\left\langle n_{\mathrm{D}}\right\rangle_{\text {add. }}$ Below $\left\langle n_{\mathrm{D}}\right\rangle_{\mathrm{cr}}$ the polymer loses D-forms, above this threshold it grows.

When the critical concentration of T-forms $\mathrm{C}_{\mathbf{c r}}(\mathrm{T})$ exceeds $\mathrm{C}_{\mathrm{cr}}(\mathrm{su})$, then the assembly proceeds spontaneously, because the free energy change $\Delta \mathrm{G}$ is less than zero; where $\mathrm{G}$ is the Gibbs free energy. The disassembly (depolymerization) of microtubules proceed spontaneously when the change of free energy is positive. Thus, the sign of the free energy determines the direction of the chemical reaction, where the critical concentration $\mathrm{C}_{\mathrm{cr}}(\mathrm{su})$ describes the dynamical equilibrium state $\Delta \mathrm{G}=0$.

The process of dynamical instability occurs when the concentration $\mathrm{C}(\mathrm{su})$ of free subunits intermediates $\mathrm{C}_{\mathbf{c r}}(\mathrm{T})$ and $\mathrm{C}_{\mathrm{cr}}(\mathrm{D})$, that is $\mathrm{C}(\mathrm{T})<\mathrm{C}(\mathrm{su})<\mathrm{C}_{\mathrm{cr}}$ (D). In case, this constraint is fulfilled then the plus end of a free microtubule grows, while the minus end shrinks, where the growth threshold and the shrinkage threshold are different, $\mathrm{C}_{\mathbf{c r}}(\mathrm{T})<\mathrm{C}_{\mathbf{c r}}(\mathrm{D})$. This means that $\mathrm{D}$-forms lean more rapidly to disassembly, while $\mathrm{T}$-forms lean more to assembly [1]. Thus, in the interphase the population of microtubules is unstable and short-lived. For example, in neural axons stable, long-lived population of microtubules replace the short-lived unstable microtubules. The disassembly of such stable structures has catastrophic consequences, axons would retract.

Thus far, the influence of the temperature on tubulin concentrations and in succession on the assembly and disassembly of microtubules was neglected. The stability of assembled microtubules is temperature-dependent. At low temperature (e.g. $4^{\circ} \mathrm{C}$ ) microtubules depolymerize into stable $\alpha, \beta$-tubulins and at the higher temperature of $37^{\circ} \mathrm{C}$ and in the presence of GTP, tubulin dimers polymerize into microtubules (growth phase) [13]. The temperature of the thermal equilibrium of $\alpha, \beta$-tubulins is settled to the mean body temperature of $37^{\circ} \mathrm{C}$. When the temperature of the thermal dynamic equilibrium distinctly descends below $37^{\circ} \mathrm{C}$, then the microtubules depolymerize and release subunits (disassembly). At higher temperature than $37^{\circ} \mathrm{C}$ the subunits repolymerize in the presence of GTP.

\section{Methods}

This contribution reverts to methods of the essential framework of quantum biology, developed in [10]. That is, the complex dynamic behavior of microtubules that oscillate between growing and shrinking phases and the initiating activities of accessory proteins are described by the approach of the non-relativistic QFT. The $\alpha$-and $\beta$-tubulin and the tubulin subunit, which compose the subunit and the group of accessory proteins represent field quanta. The essential point of this methodology is the particle interpretation, which includes their representation as matter waves, of the quantized field, where in this contribution the field quanta are Bosons. They are generated and destroyed by appropriate time-dependent creation and annihilation operators. For example, the operator $\hat{n}_{i}^{\dagger}(\mathrm{t})$ denotes the particle creation marked by the dagger symbol $\uparrow-$ in a quantum state of discrete energy level $\mathrm{E}_{\mathrm{i}}$, whereas $\hat{\mathrm{n}}_{\mathrm{i}}(\mathrm{t})$ defines the annihilation of this particle. Both operators are time dependent because the calculations are executed in the Heisenberg picture $[10,22]$. In the following all operators are marked by a "roof", so that they cannot be confused with eponymous numbers.

According to de Broglie a quantum particle with the energy $E$ is associated to the four-vector $\frac{1}{\hbar}(p, E)=(k, \omega)$, where $\hbar$ is the Planck's constant h (action quantum) divided by $2 \pi, \mathrm{k}$ is a wave vector and $\omega$ is the (circular) frequency of the associated quantum wave. The quantum mechanical wave function $\Psi(\mathrm{x}, \mathrm{t}) \sim \exp \{\mathrm{i}(\mathrm{k} \cdot \mathrm{x}-\omega \mathrm{t})\}$ of a free particle of momentum $\mathrm{p}$ and energy $\mathrm{E}$ brings in relation the two components of the four-vector. The phase velocity $\mathrm{v}_{\text {phase }}=\frac{\omega}{k}$ describes the motion of the maximum of the matter wave $\Psi$, but not the quantum velocity of a particle $\mathrm{v}_{\text {particle }}=\frac{\hbar k}{m}$. Therefore, in accordance to the particle wave duality, particles are associated with wave packets whose group velocity $\mathrm{v}_{\text {group }}=\frac{d \omega}{d k}$ is identical to the particle velocity [23].

The standard, discrete representation of the non-relativistic quantum field operators $\widehat{\Psi}$ (cell normalization) and its Hermitian conjugate are

$$
\begin{aligned}
& \widehat{\Psi}(\mathrm{x}, \mathrm{t})=\frac{1}{\sqrt{V}} \sum_{k=0}^{\infty} \hat{n}_{\mathrm{k}}(t) \exp \left\{\mathrm{i}\left(\mathrm{k} \cdot \mathrm{x}-\omega_{k} \mathrm{t}\right)\right\}, \\
& \widehat{\Psi}^{\dagger}(\mathrm{x}, \mathrm{t})=\frac{1}{\sqrt{V}} \sum_{k=0}^{\infty} \hat{n}_{k}^{\dagger}(t) \exp \left\{-\mathrm{i}\left(\mathrm{k} \cdot \mathrm{x}-\omega_{k} \mathrm{t}\right)\right\},
\end{aligned}
$$

where, for instance $\widehat{\Psi}^{\dagger}(x, \mathrm{t})$ creates a particle at location $x$ at time $\mathrm{t}$ by summation over all wave vectors $\mathrm{k}$ which includes the frequencies $\omega_{\mathrm{k}}$ of the k-dependent energy eigenstates $\left(\hbar \omega_{k}=\hbar^{2}|\mathrm{k}|^{2} / 2 \mathrm{~m}\right)$. Four relevant remarks are here indicated. First, a non-relativistic field operator contains only one annihilation resp. creation operator in contrast to relativistic field operators which are composed of creations and annihilations operators [10,22]. Second, both expansions (1) can be inverted by Fourier transformations to define $\hat{n}_{k}$ and $\hat{\mathrm{n}}_{\mathrm{k}}^{\dagger}$. Third, to simplify matters this contribution mostly restricts to the application of creation resp. annihilation operators which are associated to energy states or to locations, because they are more appropriate to the modelling of microtubules. Thus, $\hat{n}_{i}^{\dagger}$ creates a field quantum of energy $\mathrm{E}_{\mathrm{i}}=\hbar \omega_{\mathrm{i}}$ and for instance, $\hat{\alpha}_{1}^{\dagger}$ creates a $\alpha$-tubulin at site 1 . Fourth, the Bose-Einstein statistics for the quanta of this field 
are ensured by the commutation rules of Bosons [10, 22].

The dynamics of microtubule processes is represented by the definition of the interaction Hamiltonian $\widehat{\mathrm{H}}_{\text {int }}$ and the equations of motion for each individual particle operator, where every equation of motion is evaluated in the Heisenberg picture. Such an equation obeys the general form

$$
\frac{d}{d t} \widehat{\mathrm{O}}(\mathrm{t})=\frac{i}{\hbar}\left[\mathrm{H}_{\mathrm{int}}, \widehat{\mathrm{O}}(\mathrm{t})\right]-\gamma_{O} \widehat{\mathrm{O}}(\mathrm{t})+\widehat{\mathrm{F}}_{\mathrm{O}}(\mathrm{t}),
$$

where $\widehat{O}(t)$ is a time-dependent operator and $\left[\widehat{\mathrm{H}}_{\text {int }}, \widehat{\mathrm{O}}\right]=$ $\widehat{\mathrm{H}}_{\text {int }} \widehat{\mathrm{O}}-\widehat{\mathrm{O}} \widehat{\mathrm{H}}_{\text {int }}$ defines the commutator between the Hamiltonian and the operator. Further, the full equation (2) is supplemented by a damping constant $\gamma_{O}$ and by fluctuating forces $\widehat{\mathrm{F}}_{\mathrm{O}}$.

In a system of many identical (indistinguishable) particles the basis state takes the form $\left|n_{1}, n_{2}, \ldots\right\rangle$, where $n_{i}$ is the occupation number of the energetic state $E_{i}$. In case of

Bosons each of these numbers can take the integer values $\mathrm{n}_{\mathrm{i}}=0,1,2, \ldots$ Formally, these numbers are different eigenvalues of the number operators $\widehat{N}_{i}=\hat{n}_{i}^{\dagger} \widehat{n}_{i}$, where the eigenvalue equation reads $\widehat{\mathrm{N}}_{\mathrm{i}}\left|\mathrm{n}_{\mathrm{i}}\right\rangle=\mathrm{n}_{\mathrm{i}}\left|\mathrm{n}_{\mathrm{i}}\right\rangle$, with $\mathrm{i}=1$, $2, \ldots$.

In quantum systems the classical critical concentration $\mathrm{C}_{\mathrm{cr}}$ $(\mathrm{su})=\mathrm{n}_{\text {loss }}(\mathrm{su}) / \mathrm{n}_{\text {add }}(\mathrm{su})$ are replaced by the quantum version of this threshold

$$
\left\langle n_{\mathrm{su}}\right\rangle_{\mathrm{cr}}=\left\langle n_{\mathrm{su}}\right\rangle_{\text {loss }} /\left\langle n_{\mathrm{su}}\right\rangle_{\mathrm{add}} .
$$

where $\left\langle n_{\text {su }}\right\rangle$ counts the mean number of the free subunits, summarized over all energy levels $E_{i}$

$$
\left.\left\langle\mathrm{n}_{\mathrm{su}}\right\rangle=\left\langle\hat{\mathrm{n}}_{\mathrm{i}, \mathrm{su}}^{\dagger} \hat{\mathrm{n}}_{\mathrm{i}, \mathrm{su}}\right\rangle=\sum_{\mathrm{i}}<n_{\mathrm{i}, \mathrm{su}}\right\rangle=\sum_{\mathrm{i}} \frac{1}{\mathrm{e}^{\beta\left(\mathrm{E}_{\mathrm{i}}-\mu\right)}-1} .
$$

The corresponding Bose-Einstein distribution function reads

$$
\left.\mathrm{p}_{\mathrm{BE}}\left(\mathrm{n}=\mathrm{n}_{\mathrm{i}}\right)=\left(\frac{\left\langle\mathrm{n}_{\mathrm{i}}\right\rangle}{\left\langle\mathrm{n}_{\mathrm{i}}\right\rangle+1}\right)^{\mathrm{n}} \frac{1}{\left(\left\langle\mathrm{n}_{\mathrm{i}}\right\rangle+1\right)} \text { and }<\mathrm{n}_{\mathrm{i}, \mathrm{su}}\right\rangle=\sum_{n} n \mathrm{p}_{\mathrm{BE}}\left(n=\mathrm{n}_{\mathrm{i}}\right) \text {, }
$$

where the equality $n=n_{i}$ denotes the situation that $n$ particles exist at the energy level $E_{\mathrm{i}}$. Thus, the expression (4) defines the expectation value of the total number of subunits under the premise that these particles are members of a grand canonical ensemble of Bosons, which are in thermal equilibrium. The parameter $\mu$ is the chemical potential that regulates the number of particles and $\beta=1 / \mathrm{k}_{B} \breve{\mathrm{T}}$, where $\mathrm{k}_{B}$ is the Boltzmann constant and. $\breve{\mathrm{T}}$ denotes the absolute temperature (to avoid the confusion with $\mathrm{T}$-forms the temperature is marked by an inverse "roof").

\section{Solutions}

This chapter presents the equations of motion and their solutions associated to the various phases of the assembly, disassembly and dynamic instability of microtubules which occur in the course of the interphase. The cylindric form of each microtubule suggests to introduce a curved grid-based space, where the location 1 of each grid point is represented by cylinder coordinates $1 \approx\left(\mathrm{r}, \varphi_{1}, \mathrm{z}_{1}\right)$, where $\mathrm{r}$ is the constant radius, $\varphi_{\mathrm{l}}$, denotes the angle of the location 1 and $z_{1}$ the height of the position 1 .

\subsection{Polymerization of Subunits, Fluctuating Forces and Coherent Supply Rates}

The first step to assemble a protofilament, which consists of long linear string of adjacent subunits, where each subunit (heterodimer) links very tightly together the $\alpha, \beta$-tubulins monomers. In the QFT-approach $\alpha, \beta$-tubulins are represented by appropriate types of operators. The operator $\hat{\alpha}_{1}^{\dagger}(\mathrm{T})$ creates a $\alpha$-tubulin monomer at site 1 . and $\hat{\alpha}_{1}(\mathrm{~T})$ annihilates this molecule at the same position 1 . The $\alpha$-tubulin only exist in GTP-form, therefore, in the following $\alpha$-tubulin operators are denoted without the addition of $\mathrm{T}$. In analogy, the operators $\hat{\beta}_{1}^{\dagger}(\mathrm{T})$ and $\hat{\beta}_{\mathrm{l}}(\mathrm{T})$ create a $\beta$-tubulin or destroy it. The operators $\hat{\beta}_{1}^{\dagger}(\mathrm{D})$ and $\hat{\beta}_{1}(\mathrm{D})$ describe $\beta$-tubulins, where the nucleoside triphosphate hydrolyzed (GDP-form). Whereas, the hydrolysis process is $\mathrm{GTP}+\mathrm{H}_{2} \mathrm{O} \rightarrow \mathrm{GDP}+\mathrm{Ph}_{\mathrm{i}}+\mathrm{H}^{+}$, where $\mathrm{Ph}_{\mathrm{i}}$ is an inorganic phosphate group. In consequence, creation operators exist in two forms $\widehat{s u}_{1}^{\dagger}(\mathrm{T})$ and $\widehat{s u}_{1}^{\dagger}(\mathrm{D})$ and likewise the two annihilation operators $\widehat{s u}_{1}(T)$ and $\widehat{s u}_{1}(D)$.

The creation process of a subunit in T-form, describes the generation of a new heterodimer by the interaction of the two dimers with each other. This process is formally represented by the operator expression

$$
\widehat{s u}_{1}^{\dagger}(\mathrm{T}) \widehat{\alpha}_{1} \hat{\beta}_{1+1}(\mathrm{~T}) \text {. }
$$

This operator sequence defines the creation of a subunit in $\mathrm{T}$-form at the location 1 by the annihilations of an $\alpha$-tubulin at position 1 and of a $\beta$-tubulin at position $1+1$, where for convenience the subunit is identified by the position 1 . The prescribed succession of the $\alpha, \beta$-tubulins from the minus end of a protofilament is reflected by the two different positions 1 of $\alpha$-tubulin and $1+1$ of $\beta$-tubulin. Notice that the shift of the position 1 (e.g. by 1 ) is expressed in vector notation.

The similar expression for the creation of a subunit in $\mathrm{D}$ form is

$$
\widehat{\operatorname{su}}_{1}^{\dagger}(\mathrm{D}) \widehat{\alpha}_{1} \widehat{\beta}_{1+1}(\mathrm{~T}) \widehat{\mathrm{B}}_{1}^{\dagger}=\widehat{\mathrm{su}}_{1}^{\dagger}(\mathrm{D}) \widehat{\mathrm{su}}_{1}(\mathrm{~T}) \widehat{\mathrm{B}}_{1}^{\dagger}
$$

where the dissipative energy of the hydrolysis is represented by the heat-bath (reservoir) operator $\widehat{\mathrm{B}}_{1}^{\dagger}$, and the expression $\widehat{\alpha}_{1} \widehat{\beta}_{1+1}=\widehat{\mathrm{su}}_{1}(\mathrm{~T})$ represents a short form of a tubulin subunit in $\mathrm{T}$-form (7).

The local interaction Hamiltonian $\widehat{\mathrm{H}}_{\mathrm{int}, \mathrm{l}}$ for the creation of a free subunit in T-form and its reverse (Hermeticity) reads

$\widehat{\mathrm{H}}_{\mathrm{int}, \mathrm{l}}=-\frac{\hbar}{i} \mathrm{~g}\left[\widehat{\mathrm{su}}_{\mathrm{l}}^{\dagger}(\mathrm{T}) \hat{\alpha}_{1} \hat{\beta}_{\mathrm{l}+1}(\mathrm{~T})-\hat{\alpha}_{1}^{\dagger} \hat{\beta}_{1+1}^{\dagger}(\mathrm{T}) \widehat{\mathrm{su}}_{\mathrm{l}}(\mathrm{T})\right](8)$

where $\mathrm{g}$ is a real coupling constant and energy conservation is presumed (no dissipation).

The Heisenberg equations of motion for the creation operators are 


$$
\frac{d}{d t} \widehat{s u}_{1}^{\dagger}(\mathrm{T})=\mathrm{g} \hat{\alpha}_{1}^{\dagger} \widehat{\beta}_{\mathrm{l}+1}^{\dagger}(\mathrm{T})-\gamma_{\mathrm{su}} \widehat{s u}_{\mathrm{l}}^{\dagger}(\mathrm{T})+\widehat{\mathrm{F}}_{\mathrm{su}}^{\dagger}
$$

The dynamics of the subunit creation in T-form at location 1 is governed by the creation of the two composing monomers, however this composition subjects to damping and fluctuating forces.

$$
\frac{d}{d t} \hat{\alpha}_{1}^{\dagger}=-\mathrm{g} \widehat{s u}_{1}^{\dagger}(\mathrm{T}) \hat{\beta}_{1+1}(\mathrm{~T})-\gamma_{\alpha} \hat{\alpha}_{1}^{\dagger}+\hat{\mathrm{F}}_{\alpha}^{\dagger}+\hat{r}_{\alpha}^{\dagger}
$$

The dynamics of the " $\alpha$-generation" succeeds by the creation of a tubulin subunit in $\mathrm{T}$-form together with the destruction of a $\beta$-tubulin in $\mathrm{T}$-form at location $1+1$. This temporal derivative is supplemented by a damping constant $\gamma_{\alpha}$, by time-dependent fluctuating forces $\hat{\mathrm{F}}_{\alpha}^{\dagger}$ and by the supply rate $\hat{r}_{\alpha}^{\dagger}$ of $\alpha$-tubulin.

$$
\frac{d}{d t} \hat{\beta}_{1}^{\dagger}(\mathrm{T})=-\mathrm{g} \widehat{\mathrm{su}}_{1-1}^{\dagger}(\mathrm{T}) \hat{\alpha}_{1-1}-\gamma_{\beta} \hat{\beta}_{1}^{\dagger}(\mathrm{T})+\hat{\mathrm{F}}_{\beta}^{\dagger}+\hat{\mathrm{r}}_{\beta}^{\dagger} .
$$

The equation of motion of the creation of $\beta$-tubulin in Tform is associated with the creation of tubulin subunit in Tform together with the annihilation of $\alpha$-tubulin, both at the position $1-1$. This equation is again extended by damping, fluctuating forces and the supply rate $\hat{\mathrm{r}}_{\beta}^{\dagger}$ of $\beta$-tubulin. In both cases the supply rates are considered as constant coherent driving forces. The correlation of both rates is evaluated in subchapter 4.1. (ii). The equations of the equivalent annihilation operators are the Hermitian conjugates to the time derivativities of the creation operators.

To solve these three operator equations their expectation values (c-numbers) are calculated in a coherent state. This transformation unearths the physical meaning of the operator equations (9) - (11), because only these values can be compared with experimental results. The mean value of the number operator specifies the occupation number (intensity) of specific particles. Whereas, correlation functions determine the degree of coherence (dependence), which in case of molecules, can be measured for instance by frequency filters [24]. Furthermore, the supply rates (fluxes) of the $\alpha$ tubulins and $\beta$-tubulins within the cytosol can be coherent (in phase) or incoherent (out of phase). This differentiation is for quantum particles especially important, because the particles are either represented by continuous quantum coherent waves or by incoherent quantum waves caused by scattering and dephasing of the coherent waves, where a strong dephasing (damping and fluctuations) causes a hopping influx. Thus, it is instructive to differentiate between a continuous wave-like flux (coherence) and a particle-like flux (incoherence), because this difference reflects the dualism between the wave-behavior and the hopping particle-behavior.

The coupled nonlinear equations (9) - (11) are solved by the method of adiabatic elimination [10]. To apply this approach the three damping constants are cast in the following order of magnitudes $\gamma_{\beta}>\gamma_{\alpha}>\gamma_{\mathrm{su}}$. This order sequence of damping constants determines the succession of eliminations. A better clarity of the formulas is achieved by introducing the abbreviations:

$$
\hat{\mathrm{F}}_{\alpha}^{\prime}=\hat{\mathrm{F}}_{\alpha}+\hat{\mathrm{r}}_{\alpha}, \hat{\mathrm{F}}_{\beta}^{\prime}=\hat{\mathrm{F}}_{\beta}+\hat{r}_{\beta} ; \hat{\mathrm{F}}_{\alpha}^{\prime \dagger}=\hat{\mathrm{F}}_{\alpha}^{\dagger}+\hat{\mathrm{r}}_{\alpha}^{\dagger}, \hat{\mathrm{F}}_{\beta}^{\prime \dagger}=\hat{\mathrm{F}}_{\beta}^{\dagger}+\hat{\mathrm{r}}_{\beta}^{\dagger} \cdot(
$$

In the first step $\hat{\beta}_{l+1}^{\dagger}(\mathrm{T})$ is eliminated (setting the temporal derivative to zero in (11))

$$
\begin{aligned}
\hat{\beta}_{l+1}^{\dagger}(\mathrm{T}) & =-\frac{\mathrm{g}}{\gamma_{\beta}} \widehat{s u}_{1}^{\dagger}(\mathrm{T}) \hat{\alpha}_{1}+\hat{F}_{\beta}^{\prime \prime \dagger}, \text { where } \\
\hat{F}_{\beta}^{\prime \prime \dagger} & =\int_{0}^{t} e^{-\gamma_{\beta}(t-\tau)} \hat{\mathrm{F}}_{\beta}^{\prime \dagger}(\tau) d \tau .
\end{aligned}
$$

Insertion of the Hermitian conjugate to equation (13) into (10) and performing a second adiabatic elimination of $\hat{\alpha}_{l}^{\dagger}$ yields

$$
\begin{gathered}
\hat{\alpha}_{1}^{\dagger}=\left(\frac{\mathrm{g}^{2}}{\gamma_{\beta}} \widehat{s u}_{1}^{\dagger}(\mathrm{T}) \widehat{s u}_{1}(\mathrm{~T})-\gamma_{\alpha}\right)^{-1} \mathrm{~g} \widehat{s u}_{1}^{\dagger}(\mathrm{T}) \hat{\mathrm{F}}_{\beta}^{\prime}+\widehat{\mathrm{F}}_{\alpha}^{\prime \prime \dagger} \text {, where } \\
\hat{F}_{\alpha}^{\prime \prime \dagger}=\int_{0}^{t} e^{-\gamma_{\beta}(t-\tau)} \hat{\mathrm{F}}_{\alpha}^{\prime \dagger}(\tau) d \tau
\end{gathered}
$$

Insertion of the Hermitian conjugate to equation (14) into equation (13) results in

$$
\hat{\beta}_{l+1}^{\dagger}(\mathrm{T})=
$$

$$
\left.\frac{-\mathrm{g}}{\gamma_{\beta}} \widehat{s u}_{l}^{\dagger}(\mathrm{T})\left\{\left(\frac{\mathrm{g}^{2}}{\gamma_{\beta}} \widehat{s u}_{l}^{\dagger}(\mathrm{T}) \widehat{s u}_{1}(\mathrm{~T})-\gamma_{\alpha}\right)^{-1} g \widehat{s u}_{l}^{\dagger}(\mathrm{T}) \hat{\mathrm{F}}_{\beta}^{\prime \dagger}+\widehat{\mathrm{F}}_{\alpha}^{\prime}\right)\right\}+\hat{\mathrm{F}}_{\beta}^{\prime \prime \dagger} .
$$

To avoid the evaluation of further tedious calculations, we directly come to the essential results by considering only the leading term $\hat{\mathrm{F}}_{\alpha}^{\prime \dagger}$ in equation (13) and $\hat{\mathrm{F}}_{\beta}^{\prime \prime \dagger}$ in equation (15). Inserting these two dominant terms into equation (9) yields $\frac{d}{d t} \widehat{s u}_{1}^{\dagger}(\mathrm{T})=\widehat{\mathrm{G}}^{\dagger}-\gamma_{\mathrm{su}} \widehat{s u}_{l}^{\dagger}(\mathrm{T})+\widehat{\mathrm{F}}_{\mathrm{su}} ;$ with $\widehat{\mathrm{G}}^{\dagger}=\mathrm{g} \widehat{\mathrm{F}}_{\alpha}^{\prime \prime \dagger} \widehat{\mathrm{F}}_{\beta}^{\prime \prime \dagger}$

The solution of (18) reads its changed form

$$
\widehat{s u}_{1}^{\dagger}(\mathrm{T}, \mathrm{t})=\int_{0}^{t} e^{-\gamma_{s u}(t-\tau)}\left(\widehat{\mathrm{G}}^{\dagger}(\tau)+\widehat{\mathrm{F}}_{s u}^{\dagger}(\tau)\right) \mathrm{d} \tau+\widehat{s u}_{1}^{\dagger}(\mathrm{T}, 0) e^{-\gamma_{s u} \mathrm{t}}
$$

where $\widehat{s u}_{1}^{\dagger}(\mathrm{T}, 0)$ denotes the initial value.

Two special cases are worthwhile to evaluate and to compare their results, that is at first, to include only fluctuating forces (incoherent supply rate) or at second, to exclude them and only consider coherent supply rates. The next subchapter treats the first case.

(i) Fluctuating Forces
The assumption that only fluctuating forces are considered (case 1) implies that the rates in equations (10) and (11) are neglected and $\hat{\mathrm{F}}_{\alpha}^{\prime}=\hat{\mathrm{F}}_{\alpha}, \hat{\mathrm{F}}_{\beta}^{\prime}=\hat{\mathrm{F}}_{\beta}$ are set. Under these preconditions the expectation value of the number operator is calculated

$$
<\widehat{s u}_{1}^{\dagger}(\mathrm{T}, \mathrm{t}) \widehat{s u}_{1}(\mathrm{~T}, \mathrm{t})>\text {. }
$$


A typical expression that occurs during the evaluation of $\quad<\int_{0}^{t} e^{-\gamma_{s u}(t-\tau)} \widehat{\mathrm{G}}^{\dagger}(\tau) \mathrm{d} \tau \int_{0}^{t} e^{-\gamma_{s u}\left(t-\tau^{\prime}\right)} \widehat{\mathrm{G}}\left(\tau^{\prime}\right) \mathrm{d} \tau^{\prime}>$, where $(21)$ the correlation function $(20)$ is

$$
\begin{gathered}
\widehat{\mathrm{G}}^{\dagger}(\tau)=g \int_{0}^{\tau} e^{-\gamma_{\alpha}(\tau-\rho)} \hat{\mathrm{F}}_{\alpha}^{\dagger}(\rho) \mathrm{d} \rho \int_{0}^{\tau} e^{-\gamma_{\beta}\left(\tau-\rho^{\prime}\right)} \hat{\mathrm{F}}_{\beta}^{\dagger}\left(\rho^{\prime}\right) \mathrm{d} \rho^{\prime} . \\
\widehat{\mathrm{G}}\left(\tau^{\prime}\right)=g \int_{0}^{\tau^{\prime}} e^{-\gamma_{\alpha}\left(\tau^{\prime}-\sigma\right)} \hat{\mathrm{F}}_{\alpha}^{\dagger}(\sigma) \mathrm{d} \sigma \int_{0}^{\tau^{\prime}} e^{-\gamma_{\beta}\left(\tau^{\prime}-\sigma^{\prime}\right)} \hat{\mathrm{F}}_{\beta}^{\dagger}\left(\sigma^{\prime}\right) \mathrm{d} \sigma^{\prime},
\end{gathered}
$$

The result of the correlation function of $<\widehat{G}^{\dagger}(\tau) \widehat{G}\left(\tau^{\prime}\right)>$ reads

$$
\begin{gathered}
<\widehat{G}^{\dagger}(\tau) \widehat{G}\left(\tau^{\prime}\right)>=\mathrm{g}^{2}<n_{\alpha}(\check{\mathrm{T}})><n_{\beta}(\check{\mathrm{T}})>\left(1-e^{-2 \gamma_{\alpha}\left|\tau-\tau^{\prime}\right|}\right)\left(1-e^{-2 \gamma_{\beta}\left|\tau-\tau^{\prime}\right|}\right) \\
\approx \mathrm{g}^{2}<n_{\alpha}(\check{\mathrm{T}})><n_{\beta}(\check{\mathrm{T}})>
\end{gathered}
$$

where $<n_{\alpha}(\check{\mathrm{T}})>$ respectively $<n_{\beta}(\check{\mathrm{T}})>$ are the average numbers of the two free monomers $\alpha$-and $\beta$-tubulins at possibly different absolute temperatures $\breve{T}$ and the neglection of the exponential factors according to the previous

$$
\begin{gathered}
<\int_{0}^{t} e^{-\gamma_{s u}(t-\tau)} \widehat{\mathrm{G}}^{\dagger}(\tau) \mathrm{d} \tau \int_{0}^{t} e^{-\gamma_{s u}\left(t-\tau^{\prime}\right)} \widehat{\mathrm{G}}\left(\tau^{\prime}\right) \mathrm{d} \tau^{\prime}>= \\
\frac{\mathrm{g}^{2}}{\gamma_{\mathrm{su}}{ }^{2}}<n_{\alpha}(\check{\mathrm{T}})><n_{\beta}(\check{\mathrm{T}})>\left(1-e^{-\gamma_{s u} t}\right)^{2} .
\end{gathered}
$$

The calculation of (24) also requires the correlation function, for instance of $\widehat{\mathrm{F}}_{\alpha}^{\dagger}$ and $\widehat{\mathrm{F}}_{\alpha}$ which is given by

$$
<\hat{F}_{\alpha}^{\dagger}(\rho) \hat{\mathrm{F}}_{\alpha}(\sigma)>=2 \gamma_{\alpha}<n_{\alpha}(\check{\mathrm{T}})>\delta(\rho-\sigma),
$$

and equivalently

$$
<\hat{F}_{\beta}^{\dagger}\left(\rho^{\prime}\right) \hat{F}_{\beta}\left(\sigma^{\prime}\right)>=2 \gamma_{\beta}<n_{\beta}(\check{\mathrm{T}})>\delta\left(\rho^{\prime}-\sigma^{\prime}\right) .
$$

Insertion of (19) and its conjugate into (20) under consideration of (24) yields

$$
<\widehat{s u}_{1}^{\dagger}(\mathrm{T}, \mathrm{t}) \widehat{s u}_{1}(\mathrm{~T}, \mathrm{t}) \geq \frac{\mathrm{g}^{2}}{\gamma_{\mathrm{su}}^{2}}<n_{\alpha}(\check{\mathrm{T}})>\left(1-e^{-\gamma_{s u} t}\right)<n_{\beta}(\check{\mathrm{T}})>\left(1-e^{-\gamma_{s u} t}\right)+<n_{s u}(\breve{\mathrm{T}})>.
$$

The expectation value of the initial value of the subunits reads

$$
<\widehat{s u}_{\mathrm{l}}^{\dagger}(\mathrm{T}, 0) \widehat{s u}_{\mathrm{I}}(\mathrm{T}, 0)>e^{-2 \gamma_{s u} t}=<n_{s u}(\check{\mathrm{T}})>e^{-2 \gamma_{s u} t} .
$$

The intermediate expressions (22) and (23) include the correlations of the fluctuation forces which act upon the $\alpha$, $\beta$-tubulins. Thus, they demonstrate that the expected number of subunits (27), at an arbitrary site 1, depends on these fluctuation forces. But, it also depends on the damped mean numbers of the two composing proteins and the undamped mean value of the subunits. Furthermore, the composition of the heterodimers is incoherent because no coherent supply rate exists. Hence, expression (27) confirms the anticipation that the mean number of subunits is subject to a hopping process (incoherence), which is caused by damping and fluctuation processes.

The subunits and respectively the $\alpha-$ and $\beta$-tubulins are elements of different grand canonical ensembles of Bosons, thus, the mean total number of each separate ensemble of them can be calculated by formula (4). The separation into three different ensembles is required because the elements of each ensemble must be indistinguishable (identical Bosons).

Furthermore, the hopping process is clearly demonstrated by considering the variance of the Bose-Einstein distribution, for example for $\alpha$-tubulin by their variance

$$
\sigma_{\alpha}^{2}=\left\langle n_{\alpha}{ }^{2}\right\rangle-\left\langle n_{\alpha}\right\rangle^{2}=\left\langle n_{\alpha}\right\rangle\left(\left\langle n_{\alpha}\right\rangle+1\right) .
$$

The variances ${\sigma_{\beta}}^{2}$ of $\beta$-tubulins can be calculated by the same formula (29). Hence, the hopping (bunching effect) of the influx of both composing components of subunits separately occur and in consequence the polymerization of the subunits ensues discontinuity.

(ii) Coherent Supply Rates

This subchapter describes the idealized case that the fluctuating forces $\hat{\mathrm{F}}_{\alpha}$ and $\hat{\mathrm{F}}_{\beta}$ are neglected, because they generate the incoherent solutions. Thus, it is proposed that $\hat{\mathrm{F}}_{\alpha}^{\prime}=\hat{\mathrm{r}}_{\alpha}, \hat{\mathrm{F}}_{\beta}^{\prime}=\hat{r}_{\beta}$ and therefore integral (16) changes to

$$
\hat{\mathrm{F}}_{\alpha}^{\prime \prime}(t)=\int_{0}^{t} e^{-\gamma_{\alpha}(\mathrm{t}-\tau)} \hat{\mathrm{r}}_{\alpha}(\tau) \mathrm{d} \tau=\frac{\hat{\mathrm{r}}_{\alpha}(\mathrm{t})}{\gamma_{\alpha}}\left(1-e^{-\gamma_{\alpha} \mathrm{t}}\right),
$$

where $\hat{\mathrm{r}}_{\alpha}(t)=\hat{\mathrm{r}}_{\alpha}(0)$, because this rate is constant. Under the assumption that the term $e^{-\gamma_{\alpha} t}$ can be ignored and the analog term in (14) also can be neglected then the expressions (14) and (30) yield

$$
\hat{\mathrm{F}}_{\alpha}^{\prime \prime}(t)=\frac{\hat{r}_{\alpha}(\mathrm{t})}{\gamma_{\alpha}}, \hat{\mathrm{F}}_{\beta}^{\prime \prime}(\mathrm{t})=\frac{\hat{r}_{\beta}(\mathrm{t})}{\gamma_{\beta}} .
$$

Consequently, the conjugate Hermitian solution to (18) modifies to 


$$
\widehat{s u}_{1}(\mathrm{~T}, \mathrm{t})=\widehat{\mathrm{R}}_{1}(\mathrm{t})+\widehat{\mathrm{su}}_{1}(\mathrm{~T}, 0) e^{-\gamma_{s u} \mathrm{t}}+\int_{0}^{t} e^{-\gamma_{s u}(t-\tau)} \widehat{\mathrm{F}}_{s u}(\tau) \mathrm{d} \tau,
$$

where

$$
\widehat{\mathrm{R}}_{l}(\mathrm{t})=\frac{g}{\gamma_{s u} \gamma_{\alpha} \gamma_{\beta}} \hat{r}_{\alpha}(\mathrm{t}) \hat{\mathrm{r}}_{\beta}(\mathrm{t})\left(1-e^{-\gamma_{s u} t}\right)
$$

The expectation value $<\widehat{\operatorname{su}}_{1}(\mathrm{~T}, \mathrm{t})>$ coincide with $\widehat{\mathrm{R}}_{\mathrm{l}}(\mathrm{t})$, because in expression (32) the expectation value is

$$
<\widehat{s u}_{1}^{\dagger}(\mathrm{T}, \mathrm{t}) \widehat{s u}_{1}(\mathrm{~T}, \mathrm{t})>=\mathrm{R}_{1}(\mathrm{t}) \mathrm{R} *_{1}(\mathrm{t})+<\mathrm{n}_{\text {su }}\left(\mathrm{T}^{\mathrm{T}}\right)>\text {, for all positions } 1,
$$

where $\mathrm{R} *{ }_{1}$ represents the complex conjugation of $R_{1}$.

Continues coherent supply rates of $\left\langle\hat{\mathrm{r}}_{\alpha}(\mathrm{t})>\right.$ and $\left\langle\hat{\mathrm{r}}_{\beta}(\mathrm{t})>\right.$ are not guaranteed by the Bose-Einstein distribution according to the "bunching effect" (2). Such a coherent rate is ensured by the Poisson distribution

$$
p_{\text {Pois }}(n)=\frac{\left\langle n_{i, \alpha}\right\rangle^{\mathrm{n}}}{n !} e^{-\left\langle n_{i, \alpha}\right\rangle}
$$

where $\left\langle n_{\mathrm{i}, \alpha}\right\rangle$ characterizes the expectation value of $\alpha-$ tubulin of energy $E_{\mathrm{i}}$. The variance of this distribution is in contradiction to the variance of Bosons in thermal equilibrium (29)

$$
\sigma_{\alpha}^{2}=\left\langle n_{i, \alpha}{ }^{2}\right\rangle-\left\langle n_{i, \alpha}\right\rangle^{2}=\left\langle n_{i, \alpha}\right\rangle .
$$

Thus, for example the supply rate of $\alpha$-tubulin and $\beta$-tubulin in the cytosol occur with respective constant distance between the same types of molecules. Consequently, the fluxes of both monomers are continuous. With respect to the two different distribution functions (5) and (35) this change signifies a thermal non-equilibrium phase transition (dynamic equilibrium) to a changed probability function (35), which is typically observed in biological systems (e.g. influx of neurotransmitters into the synaptic cleft) or in nuclear physics (radioactive decay) and in quantum optics (coherent laser) [23].

As mentioned above, the critical concentration (threshold) of subunits $\left\langle n_{\text {su }}\right\rangle_{\text {cr }}$ (3) is the concentration of the hetrodimeric $\alpha$, $\beta$-tubulin in a dynamic equilibrium of microtubules, where the polymer is a mixture of $\mathrm{T}$-forms and $\mathrm{D}$-forms subunits [1]. The critical temperature is $\breve{\mathrm{T}}_{\mathrm{c}}=236,15^{0} \mathrm{~K}\left(37^{\circ} \mathrm{C}\right)$, where $\alpha-, \beta$ tubulins polymerize. Distinctly below $\breve{\mathrm{T}}_{\mathrm{c}}$ microtubules depolymerize, releasing $\alpha, \beta$-tubulins. The connection of the thermal control parameter $\check{\mathrm{T}}_{c}$ and the critical concentration is obvious. At the critical temperature $\breve{\mathrm{T}}_{\mathrm{c}}$ and at the critical concentration $\left\langle n_{\mathrm{su}}\right\rangle_{\mathrm{cr}}$ of subunits $(\alpha, \beta$-tubulin) a dynamical equilibrium adjusts. Below $\check{\mathrm{T}}_{\mathrm{c}}$ and below $\left\langle n_{\text {su }}\right\rangle_{\text {cr }}$ no polymerization occurs. At $\breve{\mathrm{T}}_{\mathrm{c}}$ and above the critical concentration $\left\langle n_{\text {su }}\right\rangle_{\text {cr }}$ subunits polymerize into microtubules. High temperature above $\breve{\mathrm{T}}_{\mathrm{c}}$ and for instance in case of fever $\left(38^{\circ} \mathrm{C}-42^{\circ} \mathrm{C}\right)$ and above $\left\langle n_{\mathrm{su}}\right\rangle_{\mathrm{cr}}$, the assembly of microtubules is time dependent and the microtubules elongate where both their forms and their orientations vary. Though, in cells the states far from a (stationary) thermal equilibrium the non-equilibrium phase transition is kept stable by a continuous flux of energy into the cell and by an active consumption of this energy.

After this thermodynamic "plug-in" it is appropriate to return to biological descriptions of the previously mentioned processes (e.g. assembly and disassembly of microtubules). Already the stability of the regulation of nascent tubulin may be obstructed by a degradation signal (nuclease) which binds to the tubulin molecule $[4,13]$. In this case, the supply rate of both kinds of tubulins cannot be constant because the more the mRNA is degraded, the less tubulin molecules are produced. In consequence, the regulation of stability of the RNA translation depends from the actual initial state of this translation with the result that the production rate of tubulin changes steadily and leads, for example to a wasteful (e.g. cancerous) synthesis of tubulin.

Besides the request for the stability of the regulation of the production rate of $\alpha-, \beta$-tubulins also the assembly and disassembly processes in the cytosol must be considered. So, the preferred end of a microtubule, where the assembly (addition) and disassembly (loss) occur, is the (+) end. However, their time rates are different, because the mechanism of assembly differs from that of disassembly [13]. At the distal end $(+)$ the growing rate is more slowly than the shrinking rate. Therefore, the concentration of released tubulin subunits grows during the shrinking phase and diminishes during the growth phase. Focused on the kind of the supply of free tubulin subunits, the disassembly process delivers a continuous coherent supply and the assembly process reduces the number of available tubulin subunits and the polymerization occurs stepwise.

The supply rate of free tubulin subunits is a control parameter, because it determines their concentration, which settles whether the assembly or disassembly process occur. In quantum mechanical sense this is significant for the coherence of the quantum supply rate (wave description), which depends from the continuous replenishment of particles and the sheltering against the impact of fluctuating forces. Eventually, constant supply rates are assumed, because this contribution defines a basic description of the generation of microtubules, which all possible disruptive complex processes (e.g. erroneous mRNA translation or chaotic behavior of rates of MAPs) exclude.

\subsection{Protofilament Assembly by Noise-Free Quantum Movement of Free Subunits}

Free subunits associate longitudinally to form protofilaments, where the $\alpha$-tubulins is down and the $\beta$ tubulin is up in each heterodimer. Therefore, a protofilament and a microtubule achieves a distinct structural polarity, with $\alpha$-tubulin at minus end and $\beta$-tubulin at plus end.

The basic approach of this assembly includes the transport 
of free subunits to the locations of stepwise growth of the protofilament. The sites of each integrated subunit into the originating linear, periodic structure are labeled by $1 \approx \mathrm{z}_{\mathrm{l}}$, where 1 runs from 0 till $1-1$ and $L$ defines the number of sites. The motion of a free sub unit from the location $l^{\prime}$ to the final position 1 is supported by a quantum transfer function $\operatorname{Tr}\left(l^{\prime}, 1\right)$. which conserves the energy.

The basic Hamiltonian reads

$$
\widehat{\mathrm{H}}_{\text {int }}=\hbar \sum_{l=0}^{L-1} \mathrm{~V}_{1} \widehat{\mathrm{su}}_{\mathrm{l}}^{\dagger}(\mathrm{T}) \widehat{\mathrm{su}}_{\mathrm{l}}(\mathrm{T})+\hbar \sum_{\mathrm{l}^{\prime}} \operatorname{Tr}\left(\mathrm{l}^{\prime}, \mathrm{l}\right) \widehat{\mathrm{su}}_{\mathrm{l}^{\prime} \neq 1}^{\dagger}(\mathrm{T}) \widehat{\mathrm{su}}_{\mathrm{l}}(\mathrm{T}),
$$

where $\mathrm{V}_{1}$ indicates the potential that represents the longitudinal (vertical) forces between adjacent subunits.

The equation of motion only supplemented by damping is

$$
\frac{d}{d t} \widehat{s u}_{1}^{\dagger}(\mathrm{T})=\mathrm{i} V_{1} \widehat{s u}_{1}^{\dagger}(\mathrm{T})+\mathrm{i} \sum_{\mathrm{l}^{\prime} \neq 1} \operatorname{Tr}\left(\mathrm{l}^{\prime}, \mathrm{l}\right) \widehat{s u}_{\mathrm{l}^{\prime}}^{\dagger}(\mathrm{T})-\gamma_{s u} \widehat{\mathrm{su}}_{\mathrm{l}}^{\dagger}(\mathrm{T})
$$

The solutions of (38) is accomplished by two obvious conditions, described in turn. Only nearest-neighbor transitions are allowed: $\operatorname{Tr}\left(\mathrm{l}^{\prime}, \mathrm{l}\right) \neq 0$ only for $\mathrm{l}^{\prime}=\mathrm{l} \pm \mathrm{a}$, where $\mathrm{a}$ is the distance between two adjacent subunits. That is, the position $\mathrm{l}^{\prime}$ lies in the direct vicinity of the location 1 of a protofilament. When the site 1 is already occupied then the next position $1+\mathrm{a}$ is allocated by a new free subunit. Further, the periodicity of a protofilament requires the equality

$$
\widehat{s u}_{\mathrm{l}+\mathrm{aL}}^{\dagger}=\widehat{s u}_{\mathrm{l}}^{\dagger}(\mathrm{T}) \text {. }
$$

In consequence, the following solution is proposed

$$
\begin{aligned}
& \widehat{s u}_{1}^{\dagger}(\mathrm{T})=\mathrm{S}_{1} e^{\mathrm{i}(\mathrm{kl}+\omega \mathrm{t})-\gamma_{s u} \mathrm{t}} \\
& \widehat{s u}_{\mathrm{l}}^{\dagger}(\mathrm{T})=\frac{1}{\sqrt{L}} \sum_{\mathrm{k}} c_{k} e^{i(\mathrm{kl}+\omega(\mathrm{k}) \mathrm{t})-\gamma_{s u} \mathrm{t}}=\frac{1}{\mathrm{~L}} \sum_{\mathrm{k}, \mathrm{m}} e^{i(\mathrm{k}(\mathrm{l}-\mathrm{m})+\omega(\mathrm{k}) \mathrm{t})-\gamma_{s u} \mathrm{t}} \widehat{s u}_{\mathrm{m}}^{\dagger}(\mathrm{T}, \mathrm{t}=0),
\end{aligned}
$$

with $c_{k}=\frac{1}{\sqrt{L}} \sum_{\mathrm{l}} e^{-\mathrm{ik} \mathrm{l} \widehat{s u}_{\mathrm{l}}^{\dagger}}(\mathrm{T}, \mathrm{t}=0)$, where the wave number is $\mathrm{k}=\frac{2 \pi \mathrm{n}}{\mathrm{Na}}, \mathrm{n}=0,1, \ldots, \mathrm{N}-1$, with $\mathrm{N}$ $=\mathrm{L}$.

The solution for the first potential term in equation (38) is given by (42) multiplied with the factor $\mathrm{V}_{1}$

lies in the solution (43), which is inserted. into the following correlation function

$<\phi(0)\left|\widehat{s u}_{1,}^{\dagger}\left(\mathrm{T}, \mathrm{t}^{\prime}\right) \widehat{s u}_{1}(\mathrm{~T}, \mathrm{t})\right| \phi(0)>$, with the initial condition $\Phi(0)=\widehat{s u}_{\mathrm{I}_{0}}^{\dagger}(\mathrm{T}, 0) \mid \phi_{0}>$, where $\mid \phi_{0}>$ is the vacuum state. The final result reads

$$
\begin{aligned}
& \mathrm{n}^{\prime}=\mathrm{n}-\frac{\mathrm{N}}{2}=-\frac{\mathrm{N}}{2},-\frac{\mathrm{N}}{2}+1,-\frac{\mathrm{N}}{2}+2, \ldots, 0,1,2, \ldots, \frac{\mathrm{N}}{2}-1 ; \mathrm{N} \text { even. } \\
& \text { Hence, } \cos (\mathrm{k} \mathrm{a})=\cos \left(\pi+\frac{2 \pi \mathrm{n}^{\prime}}{\mathrm{N}}\right)=-\cos \left(\frac{2 \pi \mathrm{n}^{\prime}}{N}\right), \text { where } \mathrm{k}=\frac{\pi}{\mathrm{a}}+\frac{2 \pi}{\mathrm{N} a} \mathrm{n}^{\prime}
\end{aligned}
$$

Second, linear interpolation of the cosine function

$$
-\cos \left(\frac{2 \pi \mathrm{n}^{\prime}}{\mathrm{N}}\right) \approx-1+. \frac{4\left|\mathrm{n}^{\prime}\right|}{\mathrm{N}} .
$$

Now, the revised calculation, for instance of the $\widehat{s u}_{1}^{\dagger}\left(\mathrm{T}, \mathrm{t}^{\prime}\right)$ term of the correlation function (44) is performed, where the damping factor $\gamma_{s u} \mathrm{l}^{\prime}$ and the normalization factor $1 / \mathrm{L}$ are neglected for the moment.

$$
\begin{aligned}
& \sum_{k} e^{i \mathrm{k}\left[\left(\mathrm{l}-\mathrm{l}_{0}\right)+\omega(\mathrm{k}) \mathrm{t}\right]}=\sum_{\mathrm{n}^{\prime}=-\mathrm{N} / 2}^{N / 2-1} \exp \left\{\mathrm{i} \frac{2 \pi}{\mathrm{N}}\left(\frac{N}{2}+\mathrm{n}^{\prime}\right)\left(\mathrm{l}^{\prime}-\mathrm{l}_{0}{ }^{\prime}\right)+\mathrm{i} 2 \operatorname{Tr}\left(-1+\frac{4\left|\mathrm{n}^{\prime}\right|}{N}\right) \mathrm{t}\right\} \\
& =\exp \left\{\mathrm{i} \pi\left(\mathrm{l}^{\prime}-\mathrm{l}_{0}{ }^{\prime}\right)-\mathrm{i} 2 \operatorname{Tr} \mathrm{t}\right\} \cdot \sum_{\mathrm{n} /=-\mathrm{N} / 2}^{N / 2-1} \exp \left\{\mathrm{i} \frac{2 \pi}{N}\left(\mathrm{l}^{\prime}-\mathrm{l}_{0}{ }^{\prime}\right) \mathrm{n}^{\prime}+\mathrm{i} 2 \operatorname{Tr} \frac{4\left|\mathrm{n}^{\prime}\right|}{N} \mathrm{t}\right\} .
\end{aligned}
$$

The sum over $n^{\prime}$ can be evaluated by means of the formula of finite geometric series. The explicit full result for $\widehat{s u}_{1}^{\dagger}(\mathrm{T})$ 
reads

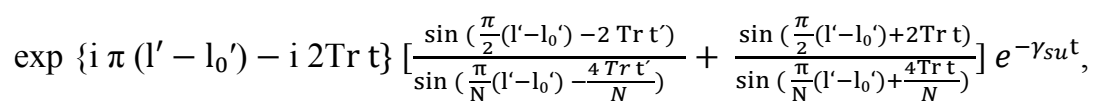

where the preceding modulation factor initiates strong oscillations. Both terms describe wave packets (quantum matter waves) which moves in opposite directions; where the first wave packet runs towards the cytosol and the second wave packet backwards. Thus, the sum of both expressions is a superposition (quantum interference) of two counterrunning wave packets with group velocities $\mathrm{v}_{\text {group }}=\frac{d \omega}{d k}$. The maximum of both wave packets lies at zero; more precise, where the arguments of the sinus functions of the nominators and denominators disappear. The peak height is N/2. Both maxima run with the phase velocity $\mathrm{v}_{\text {phase }}=\frac{\omega}{k}$. The behavior of $\widehat{s u}_{1}(\mathrm{~T})$ is equivalent to that of $\widehat{\operatorname{su}}_{1}^{\dagger}(\mathrm{T})$, up to the complex conjugation of the modulation factor. Hence, the approximation of the correlation factor (44) is given by the multiplication of $\widehat{s u}_{1}^{\dagger}(\mathrm{T})$ with $\widehat{s u}_{1}(\mathrm{~T})$.

The next step would be to find the solution of the complete equation (38), where the fluctuating forces are included. However, the impact of quantum fluctuations was already demonstrated in subchapter 4.1. (i), therefore only the wellknown effect of fluctuations is here mentioned. The coherent part of the solution is destroyed by fluctuating forces. and only the incoherent part of the solution remains. Only, when the coherent part is sheltered against the impact of fluctuations then the coherent solution will be not destroyed. This effect is observed in the case of superconductivity. However, even in biological systems (receptors) the hypothesis was proposed that human consciousness can protect coherent solutions against environmental influences [25].

\subsection{Nucleation of a Microtubule on MTOC}

The contribution of this chapter is devoted to the nucleation of a first lightly inclined loop of D-form subunits on top on a $\gamma$-tubulin ring at the minus end, which is mediated by accessory proteins. These proteins are necessary for initiating the assembling of microtubules at the minus, where they bind on the MTOC whose pericentriolar arranged material is an ordered lattice.

The most important accessory protein is the $\gamma$-tubulin $(\gamma$ TuRC, tubulin ring complex), which remains associated with the minus end, where a ring of $\gamma$ tubulin serves as a template which creates an inclined loop of a microtubule even at subcritical concentrations. After assembly of the tubulin subunits the hydrolyzation of the GTP the changed conformation of GDP forces the protofilament into a curved shape (lateral forces). Hereby, much of the diphosphate bond energy is stored in the polymer, where the remaining energy is released (dissipates). This prosses of the release of free energy is represented by the heat bath operator $\widehat{\mathrm{B}}_{1}^{\dagger}$.

The discrete cylindrical coordinates $1=\left(r, \varphi_{1}, z_{1}\right)$, with a fixed inner radius of about $\mathrm{r}=14 \mathrm{~nm}$ or a fixed outer radius of ca. $24 \mathrm{~nm}$, describe each location of a tubulin subunit within the loops of a microtubule, which spirals upwards from the first loop that emanates from the MTOC. Hence, in contrary to the previous linear coordinates of a protofilament, now cylindrical coordinates are applied to describe each position of a tubulin subunit within a microtubule. The angle $\varphi$ runs stepwise from zero to $2 \pi$ with the difference of $\Delta \varphi_{1}$, $=.5^{\circ}$ between 1 and $1+1$ of two adjacent horizontal tubulin subunits. The total height of a microtubule varies between 1 $\mu \mathrm{m}$ till some hundred $\mu \mathrm{m}$. The height of a tubulin subunit is 8 $\mathrm{nm}$, [13]. When the length of a microtubule is $1 \mu \mathrm{m}$ then 125 tubulin subunits compose a protofilament (usually 13 protofilaments compose a microtubule), where the distance (distance of their barycenter's) between two vertical locations of tubulin subunits which directly stack over one another is $\Delta$ $\mathrm{z}_{1}=4 \mathrm{~nm}$.

In topological notation each location within a microtubule can be described with relation to each position of the basic loop, which is path homotopic to a circle $S^{1}$ $=\left\{e^{\mathrm{ix}} ; \mathrm{x}\right.$ is real; $\left.\left|e^{\mathrm{ix}}=1\right|\right\}$. Further, the circle is an element of the first homotopy group $\pi_{1}$ which again is homeomorphic $(\sim)$ to the natural numbers; $\pi_{1}\left(S^{1}\right) \sim \mathbb{Z}[26]$. This means more descriptively that a microtubule can be represented by a cylindrical helix of fibers (vertical lines) above $S^{1}$, where each point of a fiber is characterized by natural number. The set of all equivalent points on a fiber which lies along the same vertical line are projected onto the same point $\mathrm{x}$ of $\mathrm{S}^{1}$. In the context of symmetry considerations, it is worthwhile to mention that $S^{1}$ is homeomorphic to the unitary group $U(1): S^{1} \sim U(1)$, where both groups are Lie groups. Thus, the shrinkage of a microtubule brakes the U (1) symmetry.

Now back to the first position of the loop of a microtubule (minus end) which is built up by a column of three composing molecules with a fixed angle $\varphi_{1}$, and varying heights $z_{1}$. According to the tininess of $\Delta z_{1}$ and to the mathematical simplification of the local Hamiltonian the different locations of the three vertical aligned composing molecules are encapsulated to one location 1.

Thus, the local interaction Hamiltonian reads

$$
\widehat{\mathrm{H}}_{i n t, \mathrm{I}}=\hbar \mathrm{g}_{1}\left(\widehat{\gamma}_{1}^{\dagger} \widehat{\mathrm{ac}}_{1} \widehat{\mathrm{su}}_{1}^{\dagger}(\mathrm{D}) \widehat{\mathrm{su}}_{1}(\mathrm{~T}) \widehat{\mathrm{B}}_{1}^{\dagger}+\widehat{\mathrm{su}}_{1}^{\dagger}(\mathrm{T}) \widehat{\mathrm{su}}_{1}(\mathrm{D}) \widehat{\mathrm{ac}}_{1}^{\dagger} \widehat{\gamma}_{1} \widehat{\mathrm{B}}_{1}\right),
$$

where $g_{1}$ is a real coupling constant. The first term of this Hamiltonian creates a $\gamma$-tubulin molecule on top of an accessory protein (MAP), which is annihilated $\left(\widehat{a c}_{1}\right)$. On top of a $\gamma$-tubulin molecule a subunit in D-form is created and a subunit in T-form is annihilated. The hydrolysis of GTP is denoted by a heat bath (reservoir) $\widehat{\mathrm{B}}_{1}^{\dagger}$, which describes the release of free energy (dissipation). The diphosphate remains trapped (GDP) in the loop structure and forces it into a 
curved shape (lateral forces). The attending release of inorganic phosphate is here not relevant and hence omitted. It will be later on considered when the shrinking phase will be described in (4.4.2.). The second term of (50) represents the Hermitian conjugate of the first term.

The total Hamiltonian (complete basic loop) is

$$
\widehat{\mathrm{H}}_{i \mathrm{nt}}=\sum_{l} \widehat{\mathrm{H}}_{\mathrm{int}, \mathrm{l}}, \text { where } \mathbf{l} \approx\left(\varphi_{\mathrm{l}}, \mathrm{z}_{\mathrm{l}}\right)
$$

The complete set of local Heisenberg equations of motion resulting from the Hamiltonian (50), which are only augmented by damping constants, are given by

$$
\begin{aligned}
& \frac{d}{d t} \widehat{\operatorname{su}}_{\mathbf{l}}^{\dagger}(\mathrm{T})=\mathrm{i} \mathrm{g}_{1} \widehat{\gamma}_{\mathbf{l}}^{\dagger} \widehat{\mathrm{ac}}_{\mathbf{l}} \widehat{\mathrm{su}}_{\mathbf{l}}^{\dagger}(\mathrm{D}) \widehat{\mathrm{B}}_{\mathrm{l}}^{\dagger}-\gamma_{\mathrm{suT}} \widehat{\mathrm{su}}_{\mathbf{l}}^{\dagger}(\mathrm{T}) \text {, } \\
& \frac{d}{d t} \widehat{\mathrm{su}}_{\mathrm{l}}^{\dagger}(\mathrm{D})=\mathrm{i} \mathrm{g}_{1} \widehat{\gamma}_{\mathrm{l}} \widehat{\mathrm{ac}}_{\mathrm{l}}^{\dagger} \widehat{\mathrm{su}}_{\mathrm{l}}^{\dagger}(\mathrm{T}) \widehat{\mathrm{B}}_{\mathrm{l}}-\gamma_{\mathrm{suD}} \widehat{\mathrm{su}}_{\mathrm{l}}^{\dagger}(\mathrm{D}), \\
& \frac{d}{d t} \widehat{\gamma}_{1}^{\dagger}=\mathrm{i} \mathrm{g}_{1} \widehat{\mathrm{ac}}_{1}^{\dagger} \widehat{s u}_{1}(\mathrm{D}) \widehat{\mathrm{su}}_{1}^{\dagger}(\mathrm{T}) \widehat{\mathrm{B}}_{1}-\gamma_{\gamma} \widehat{\gamma}_{1}^{\dagger} \text {, } \\
& \frac{d}{d t} \widehat{\mathrm{ac}}_{\mathrm{I}}^{\dagger}=\mathrm{i} \mathrm{g}_{1} \widehat{\gamma}_{\mathrm{l}}^{\dagger} \widehat{\mathrm{su}}_{\mathrm{l}}^{\dagger}(\mathrm{D}) \widehat{\mathrm{su}}_{\mathrm{l}}(\mathrm{T}) \widehat{\mathrm{B}}_{\mathrm{l}}^{\dagger}-\gamma_{\mathrm{ac}} \widehat{\mathrm{ac}}_{\mathrm{l}}^{\dagger} \text {, } \\
& \frac{d}{d \mathrm{t}} \widehat{\mathrm{B}}_{1}^{\dagger}=\mathrm{ig} \widehat{\gamma}_{1} \widehat{\mathrm{ac}}_{1}^{\dagger} \widehat{\mathrm{su}}_{1}^{\dagger}(\mathrm{T}) \widehat{\mathrm{su}}_{1}^{\dagger}(\mathrm{D})-\gamma_{\mathrm{B}} \widehat{\mathrm{B}}_{1}^{\dagger} \text {. }
\end{aligned}
$$

To elaborate stepwise the solutions the following modifications are appropriate. The first assumption concerns the damping constants of the accessory protein and of the $\gamma$ tubulin, where both are set to zero $\gamma_{\mathrm{ac}}=\gamma_{\gamma}=0$. The second simplification pertains the B-field, which is set to 1 in the equations (52) till (55). The solutions of equation (56) are described in [10]. All these modifications make it possible to elaborate analytical solutions of the differential equations (52) till (55).

The remaining four operator equations are replaced by their expectation values in a coherent state representation, whereby they become c-numbers. Thus, the two proposed replacements for the solutions of the GTP and GDP forms of tubulin are:

$$
\widehat{\mathrm{su}}_{1}^{\dagger}(\mathrm{T})=\sin \mathrm{f}(\mathrm{t}) e^{-\gamma \mathrm{t}}, \widehat{\mathrm{su}}_{1}^{\dagger}(\mathrm{D})=\cos \mathrm{f}(\mathrm{t}) e^{-\gamma \mathrm{t}},
$$

where it is assumed that $\gamma_{\text {suT }}=\gamma_{\text {suG }}=\gamma$. The initial conditions for the subunits are $\left.\widehat{\operatorname{su}}_{1}^{\dagger}(\mathrm{T}, 0)\right)=0, \widehat{\operatorname{su}}_{1}^{\dagger}(\mathrm{D}, 0)=$ 1. The ansatz for the $\gamma$-tubulin and the accessory protein read

$$
\hat{\gamma}_{1}^{\dagger}=\cos \mathrm{G}(\mathrm{t}), \widehat{a c}_{1}^{\dagger}=\mathrm{i} \sin \mathrm{G}(\mathrm{t}),
$$

where their initial conditions are $\hat{\gamma}_{1}^{\dagger}(0)=1, \widehat{a c}_{1}^{\dagger}(0)=0$.

Insertion of these four proposed solutions into the corresponding equations (52) till (55) yield the two coupled equations for $\mathrm{f}$ and $\mathrm{G}$ :

$$
\begin{gathered}
\frac{d f}{d \mathrm{t}}=\frac{1}{2} \mathrm{~g}_{1} \sin (2 \mathrm{G})=\mathrm{g}_{1} \sin (\mathrm{G}) \cos (\mathrm{G}) . \\
\frac{d G}{d \mathrm{t}}=\frac{1}{2} \mathrm{~g}_{1} \sin (2 \mathrm{f}) e^{-\gamma \mathrm{t}}=\mathrm{g}_{1} \sin (\mathrm{f}) \cos (\mathrm{f}) e^{-\gamma \mathrm{t}} .
\end{gathered}
$$

The first impression of the type of the solutions can be obtained when the damping constant $\gamma$ is switched off. Then the symmetry of the two equations (59) and (60) immediately suggest to set $f= \pm G$. Thus, by separation of variables the modified equation (60) becomes the form

$$
\frac{d G}{\sin (\mathrm{G}) \cos (\mathrm{G})}= \pm \mathrm{g}_{1} \mathrm{dt}
$$

The integral of (61) reads

$$
\ln \operatorname{tg}\left(\mathrm{G}(\mathrm{t})-\ln \operatorname{tg} \mathrm{G}\left(\mathrm{t}_{0}\right)= \pm \mathrm{g}_{1}\left(\mathrm{t}-\mathrm{t}_{0}\right),\right.
$$

which is equivalent to $\operatorname{tg} \mathrm{G}(\mathrm{t})=\operatorname{tg} \mathrm{G}\left(\mathrm{t}_{0}\right) e^{ \pm \mathrm{g}_{1}\left(\mathrm{t}-\mathrm{t}_{0}\right)}$.

The solution for $\mathrm{f}(59)$ is obviously

$$
f(t)=g_{1} \int_{t_{0}}^{t} g_{1} \sin G(\tau) \cos G(\tau) d \tau+f\left(t_{0}\right)
$$

It is revealing to consider the $\pi$-periodic, symmetric phase space which is spanned between $\mathrm{f}$ ( $\mathrm{x}$-axis) and $\mathrm{G}$ (y-axis). The phase portrait ( $G$ versus $f$ ), which is numerically calculated (e.g. $g_{1}=0.1$ ), show centers at different middle points, which are horizontally and vertically separated by the distance of $\pi$ [10]. The set of the corresponding orbits starts at predefined initial points and turns around each central point (e.g. anticlockwise that corresponds to the minus sign), where their phase flow is restricted to a quadrate of an edge length of a bit less than $\pi / 2$. Thus, the bundle of trajectories shows orbits that are classical circles $S^{1}$ and path homotopical equivalent circles in the vicinity of the borderlines.

In the next step the damping constant is switched on, where $\gamma \ll \mathrm{g}_{1}$ (e.g. $\gamma=001, \mathrm{~g}_{1}=0.1$ ) is assumed. As a consequence, the phase flow in the same phase space $(G$ versus f) changes dramatically. All "circles" dissolve and the changed orbits run away (+sign) from the centers or direct to the centers (- sign). So, in this case the + sign must be excluded because the "runaway" solution is for an assembly process inappropriate. For a disassembly process it is indicated. In addition, the previously observed U (1) symmetry, which is characteristic for electromagnetic interactions, is spontaneously broken. This effect shows some formally resemblance to the astonishing topological turbulence described in [20]. But, this similarity is not a biological resemblance because the mentioned paper describes the influence ("bombardment") of Rho-GTPs on cell membranes which generates unstable vortices.

\subsection{Growths and Shrinkage of Microtubules; the Dynamic Instability}

The changeover between slow growth and rapid shrinkage of a microtubule is a phenomenon that is called dynamic instability. This change of phases occurs within some minutes. A growing microtubule has T-forms on its plus end which form a DTP-cap. When this cap is lost then the microtubule begins to shrink, this event is called "catastrophe". The event, where the microtubule growth resumes is called "rescue".

The assembly of microtubule is performed by adding $\mathrm{T}$ form subunits on the plus end, because the most microtubules associate with their minus end to MTOCs. Thus, the descriptions of this chapter concentrate to the plus end and to concentrations of the free subunits $\mathrm{C}$ (su) that lie in the 
intermediate position $\mathrm{C}_{\mathrm{cr}}(\mathrm{T})<\mathrm{C}(\mathrm{su})<\mathrm{C}_{\mathbf{c r}}(\mathrm{D})$ and remains there. When the polymerization (assembly) rate) of $\mathrm{T}$-forms is greater than the dissociation (disassembly) rate which is initiated by the hydrolysis of GTP, then a GTP-cup grows on top of a GDP microtubule. When the polymerization rate is lower than the hydrolysis rate then the GTP-cup changes to a GDP-cap, which splays and gives the plus end a frayed appearance ("catastrophe"). However, before a shrinking microtubule vanishes GDP-GTP exchanges occur, where a GTP tubulin is set free and creates the self-assembly on top of the plus end ("rescue").

$$
\left.\widehat{\mathrm{H}}_{i n t, 1}=\hbar \mathrm{g}_{2}\left(\widehat{\mathrm{su}}_{1}^{\dagger}(\mathrm{T}) \widehat{\mathrm{su}}_{1}(\mathrm{D}) \widehat{\mathrm{ac}}_{1}^{\dagger} \widehat{\mathrm{pk}}_{1}+\widehat{\mathrm{pk}}_{1}^{\dagger} \widehat{\mathrm{ac}}_{1} \widehat{\mathrm{su}}_{1}^{\dagger}(\mathrm{D}) \widehat{\mathrm{su}}_{1}(\mathrm{~T})\right)+\hbar \mathrm{g}_{3}\left(\widehat{\mathrm{su}}_{1+1}^{\dagger}(\mathrm{D}) \widehat{\mathrm{su}}_{1}(\mathrm{~T})\right) \widehat{\mathrm{B}}_{1+1}^{\dagger}+\widehat{\mathrm{su}}_{1}^{\dagger}(\mathrm{T}) \widehat{\mathrm{su}}_{1+1}(\mathrm{D}) \widehat{\mathrm{B}}_{1+1}\right)
$$

where $\mathrm{g}_{2}$ and $\mathrm{g}_{3}$ are real coupling constants.

The first term describes the creation of a GTP-tubulin by a GDP-GTP exchange $\left(\widehat{\mathrm{su}}_{1}^{\dagger}(\mathrm{T}) \widehat{\mathrm{su}}_{1}(\mathrm{D})\right)$, which occurs when the D-Form depolymerizes at location 1 . Hence this term describes the generation of a T-form (e.g. GTP-cap) at location 1. The creation of an accessory protein $\left(\widehat{\mathrm{ac}}_{1}^{\dagger}\right)$ is supported by the phosphorylation of the protein kinase $\left(\widehat{\mathrm{pk}}_{\mathrm{l}}\right)$, whereby free energy is released (ATP-ADP exchange), which is here neglected, because the focus lies in the hydrolysis. The protein kinase is an intracellular signaling protein that acts as a molecular switch. The accessory protein is for example, the XMAP215 which accelerates the assembly of a T-form.

The second term represents the Hermitian conjugate of the first term (inverse process), where the standard change of a $\mathrm{T}$-form (hydrolysis) into a D-form is expressed by $\widehat{\mathrm{su}}_{1}^{\dagger}$ (D) $\left.\widehat{s u}_{1}(\mathrm{~T})\right)$. Furthermore, the enzyme protein kinase is created $\left(\widehat{\mathrm{pk}}_{1}^{\dagger}\right)$ and XMAP215 is annihilated $\left(\widehat{\mathrm{ac}}_{1}\right)$. Thus, the second term describes the generation of a D-form (GDP-cap) at the location 1 of the plus end.

The third term of (64) deals with the allocation of a D-

\subsubsection{Growth of the Plus End of a Microtubule}

The basic local interaction Hamiltonian describes the allocation for example, of two D-forms at the sequential horizontal positions 1 and $1+1$ of the plus end of a less stable GDP microtubule, where for instance the GDP-GTP exchange ("rescue") can occur or non-hydrolyzed T-form exist. This approach has the key advantage that the Hamiltonian for the shrinkage of microtubules remains with only minor changes (4.4.2.). The Hamiltonian reads
Form at the next position $1+1\left(\widehat{s u}_{1+1}^{\dagger}(\mathrm{D})\right)$, which is supported by the annihilation of a T-form (hydrolysis) at the previous position $1\left(\widehat{s u}_{1}(\mathrm{~T})\right)$ ), where free energy is released $\left(\widehat{\mathrm{B}}_{1+1}^{\dagger}\right)$. That is, the released free energy of the hydrolysis of the T-Form at location 1 supplies the allocation of a D-form at location $1+1$. Thus, this term takes up again the topic of the process described by the second term and concentrates essentially to the energetic aspect of the GTP hydrolysis.

The fourth term describes the depolymerization of the DForm at location 1+1, which causes the creation of a T-Form at position 1 and the consumption of the released energy $\left(\widehat{\mathrm{B}}_{1+1}\right)$.

The total Hamiltonian describes the complete horizontal loop of the plus end, where for reason of generality (Hermeticity) both kind of caps are integrated.

$$
\widehat{\mathrm{H}}_{i \mathrm{nt}}=\sum_{l} \widehat{\mathrm{H}}_{\mathrm{int}, \mathrm{l}}, \text { where } \mathrm{l} \approx\left(\varphi_{\mathrm{l}} . \mathrm{z}_{\mathrm{l}}\right) .
$$

The complete set of local Heisenberg equations of motion corresponding to the Hamiltonian (64) is only supplemented by damping constants, as in chapter 4.3 :

$$
\begin{aligned}
& \frac{d}{d t} \widehat{\operatorname{su}}_{1}^{\dagger}(\mathrm{T})=\mathrm{ig} \widehat{\mathrm{su}}_{1}^{\dagger}(\mathrm{D}) \widehat{\mathrm{pk}}_{1}^{\dagger} \widehat{a c}_{1}+\mathrm{i} \mathrm{g}_{3} \widehat{\mathrm{su}}_{\mathbf{l}+\mathbf{1}}^{\dagger}(\mathrm{D}) \widehat{\mathrm{B}}_{\mathbf{1}+\mathbf{1}}^{\dagger}-\gamma_{\mathrm{suT}} \widehat{\mathrm{su}}_{\mathrm{l}}^{\dagger}(\mathrm{T}) \\
& \frac{d}{d t} \widehat{\operatorname{su}}_{1}^{\dagger}(\mathrm{D})=i \mathrm{~g}_{2} \widehat{\mathrm{su}}_{\mathrm{l}}^{\dagger}(\mathrm{T}) \widehat{\mathrm{ac}}_{1}^{\dagger} \widehat{\mathrm{pk}}_{1}+i \mathrm{~g}_{3} \widehat{\mathrm{su}}_{\mathrm{l}-1}^{\dagger}(\mathrm{T}) \widehat{\mathrm{B}}_{1}-\gamma_{\mathrm{suD}} \widehat{\mathrm{su}}_{1}^{\dagger}(\mathrm{D}), \\
& \frac{d}{d t} \widehat{\mathrm{pk}}_{\mathrm{l}}^{\dagger}=\mathrm{i} \mathrm{g}_{2} \widehat{\mathrm{su}}_{\mathrm{l}}(\mathrm{D}) \widehat{\mathrm{ac}}_{\mathrm{I}}^{\dagger} \widehat{\mathrm{su}}_{\mathrm{l}}^{\dagger}(\mathrm{T})-\gamma_{\mathrm{pk}} \widehat{\mathrm{pk}}_{\mathrm{l}}^{\dagger} \text {, } \\
& \frac{d}{d t} \widehat{\mathrm{ac}}_{\mathbf{l}}^{\dagger}=\mathrm{i} \mathrm{g}_{2} \widehat{\mathrm{su}}_{\mathbf{l}}^{\dagger}(\mathrm{D}) \widehat{\mathrm{pk}}_{\mathrm{l}}^{\dagger} \widehat{\mathrm{su}}_{\mathbf{l}}(\mathrm{T})-\gamma_{\mathrm{ac}} \widehat{\mathrm{ac}}_{\mathbf{l}}^{\dagger} \\
& \frac{d}{d t} \widehat{\mathrm{B}}_{\mathrm{l}}^{\dagger}=\mathrm{i} \mathrm{g}_{3} \widehat{\mathrm{su}}_{\mathrm{l}-\mathbf{1}}^{\dagger}(\mathrm{T}) \widehat{\mathrm{su}}_{\mathrm{l}}(\mathrm{D})-\gamma_{\mathrm{B}} \widehat{\mathrm{B}}_{\mathrm{l}}^{\dagger} \text {. } \\
& \frac{d}{d t} \widehat{\mathrm{su}}_{\mathrm{I}}^{\dagger}(\mathrm{D})=\mathrm{i} \mathrm{g}_{2} \widehat{\mathrm{su}}_{\mathrm{I}}^{\dagger}(\mathrm{T}) \widehat{\mathrm{ac}}_{\mathrm{I}}^{\dagger} \widehat{\mathrm{pk}}_{\mathrm{I}}-\gamma_{\mathrm{suD}} \widehat{\mathrm{su}}_{\mathrm{l}}^{\dagger}(\mathrm{D}) \\
& \frac{d}{d t} \widehat{\mathrm{pk}}_{1}^{\dagger}=\mathrm{i} \mathrm{g}_{2} \widehat{\mathrm{su}}_{1}(\mathrm{D}) \widehat{\mathrm{ac}}_{1}^{\dagger} \widehat{\mathrm{su}}_{1}^{\dagger}(\mathrm{T}) \\
& \frac{d}{d t} \widehat{\mathrm{ac}}_{1}^{\dagger}=\mathrm{i} \mathrm{g}_{2} \widehat{s u}_{1}^{\dagger}(\mathrm{D}) \widehat{\mathrm{pk}}_{1}^{\dagger} \widehat{\mathrm{su}}_{\mathrm{l}}(\mathrm{T}) \text {. }
\end{aligned}
$$

In a first approach the solution for the initial location 1 (e.g. $1=1$ ) are simplified by the following assumptions. First, all terms in equations (66) till (70) which contain the prospective location $1+1$ or the previous location $1-1$ can be deleted, since these states actually does not exist. Second, both damping constants in (68) and in (69) are set to zero $\gamma_{\mathrm{pk}}=\gamma_{\mathrm{ac}}=0$. The resulting reduced equations are:

$$
\frac{d}{d t} \widehat{\mathrm{su}}_{\mathrm{l}}^{\dagger}(\mathrm{T})=\mathrm{i} \mathrm{g}_{2} \widehat{\mathrm{su}}_{\mathrm{l}}^{\dagger}(\mathrm{D}) \widehat{\mathrm{pk}}_{\mathrm{l}}^{\dagger} \widehat{\mathrm{ac}}_{\mathrm{l}}-\gamma_{\mathrm{suT}} \widehat{\mathrm{su}}_{\mathrm{l}}^{\dagger}(\mathrm{T}),
$$

When finally, the operator $\widehat{\mathrm{pk}}_{1}^{\dagger}$ in equations (71) till (74) is replaced by the operator $\hat{\gamma}_{1}^{\dagger}$ in equations (52) till (55), then both sets of equations looks equally. This conformity is not accidental, because the Hamiltonian defined in equation (50) and the first line of the Hamiltonian presented in (64) describe both the elementary assembly process of a microtube without consideration of the energetic aspect. The second line of the Hamiltonian (64) qualifies the conservation of energy. In consequence, the equations of motion (71) till (74) can also be solved analytically by the same approach as it was done in 
chapter 4.3. To avoid redundancy, the same solution method will not be repeated here.

Instead the endeavor goes back to the evaluation of the solutions of equations (66) till (69) at the next position $1+1$, where 1 is considered as the initial locations or equivalently 1
$1=2$, if the initial position is $1=1$. The dynamics of the $\mathrm{B}$ field operator is omitted (70), because its solution is tedious and needs a separate solution ansatz as the four remaining equations [3]. The set of the actualized Heisenberg equations at the location $1+1$ reads

$$
\begin{aligned}
& \frac{d}{d t} \widehat{\mathrm{su}}_{\mathrm{l}+1}^{\dagger}(\mathrm{D})=\mathrm{i} \mathrm{g}_{2} \widehat{\mathrm{su}}_{\mathrm{l}+1}^{\dagger}(\mathrm{T}) \widehat{\mathrm{ac}}_{\mathrm{l}+1}^{\dagger} \widehat{\mathrm{pk}}_{\mathrm{l}+1}+\mathrm{i} \mathrm{g}_{3} \widehat{\mathrm{su}}_{\mathrm{l}}^{\dagger}(\mathrm{T}) \widehat{\mathrm{B}}_{\mathrm{l}+1}-\gamma_{\mathrm{suD}} \widehat{\mathrm{su}}_{\mathrm{l}+1}^{\dagger}(\mathrm{D}), \\
& \frac{d}{d t} \widehat{\mathrm{su}}_{\mathrm{l}+1}^{\dagger}(\mathrm{T})=\mathrm{i} \mathrm{g}_{2} \widehat{\mathrm{su}}_{\mathrm{l}+1}^{\dagger}(\mathrm{D}) \widehat{\mathrm{pk}}_{\mathrm{l}+1}^{\dagger} \widehat{\mathrm{ac}}_{\mathrm{l}+1}+\mathrm{i} \mathrm{g}_{3} \widehat{\mathrm{su}}_{\mathrm{l}+2}^{\dagger}(\mathrm{D}) \widehat{\mathrm{B}}_{\mathrm{l}+2}^{\dagger}-\gamma_{\mathrm{suT}} \widehat{\mathrm{su}}_{\mathrm{l}+1}^{\dagger}(\mathrm{T}), \\
& \frac{d}{d t} \widehat{\mathrm{pk}}_{\mathrm{l}+1}^{\dagger}=\mathrm{ig} \widehat{\mathrm{su}}_{\mathrm{l}+1}(\mathrm{D}) \widehat{\mathrm{ac}}_{\mathrm{l}+1}^{\dagger} \widehat{\mathrm{su}}_{\mathrm{l}+1}^{\dagger}(\mathrm{T}) \text {. } \\
& \frac{d}{d t} \widehat{a c}_{1+1}^{\dagger}=\mathrm{i} \mathrm{g}_{2} \widehat{s u}_{\mathrm{l}+1}^{\dagger}(\mathrm{D}) \widehat{p k}_{\mathrm{l}+1}^{\dagger} \widehat{s u}_{1+1}(\mathrm{~T}) \text {. }
\end{aligned}
$$

The expression $\operatorname{ig}_{3} \widehat{\mathrm{su}}_{\mathrm{l}+2}^{\dagger}$ (D) $\widehat{\mathrm{B}}_{\mathrm{l}+2}^{\dagger}$ in (75) can be neglected, because this state is not yet created. However, the term $\operatorname{ig}_{3} \widehat{\operatorname{su}}_{1}^{\dagger}(\mathrm{T}) \widehat{\mathrm{B}}_{1+1}$ in (76) still exists. Furthermore, in this sub-chapter hopping (incoherent) solutions are presented. In the next sub-chapter coherent wave solutions are presented.

The proposed ansatz to solve the two first differential equations (75) and (76) is facilitated by the introduction of the following abbreviations:

$$
\mathrm{X}_{1}, \mathrm{X}_{4}=\mathrm{g}_{2} \widehat{\mathrm{ac}}_{\mathrm{l}+1}^{\dagger} \widehat{\mathrm{pk}}_{\mathrm{l}+1},
$$

where $\mathrm{X}_{4}$ is real.

Insertion of these variables into the equations (75) and (76) reformulate them to

$$
\begin{gathered}
\frac{d}{d t} \mathrm{X}_{1}=-\gamma \int_{t_{1}}^{t} e^{-\gamma(\mathrm{t}-\tau)} \cos \left(\mathrm{X}_{5}(\mathrm{t})-\mathrm{X}_{5}(\tau)\right) \mathrm{X}_{3}(\tau) \mathrm{d} \tau+\mathrm{X}_{3}(\mathrm{t}) e^{-\gamma(\mathrm{t}-\mathrm{t})} \cos \left(\mathrm{X}_{5}(\mathrm{t})-\mathrm{X}_{5}(\mathrm{t})\right) \\
-\frac{d}{d t} \mathrm{X}_{5} \int_{t_{1}}^{t} e^{-\gamma(\mathrm{t}-\tau)} \sin \left(\mathrm{X}_{5}(\mathrm{t})-\mathrm{X}_{5}(\tau)\right) \mathrm{X}_{3}(\tau) \mathrm{d} \tau=-\gamma \mathrm{X}_{1}-\mathrm{X}_{3}\left(-\mathrm{i} \mathrm{X}_{2}\right)+\mathrm{X}_{3}=i \mathrm{X}_{2} \mathrm{X}_{3}+\mathrm{X}_{3}-\gamma \mathrm{X}_{1} .
\end{gathered}
$$

Rewritten in original forms the two solutions read

$$
\begin{gathered}
\widehat{s u}_{1+1}^{\dagger}(\mathrm{D})=\int_{t_{1}}^{t} \mathrm{I}_{D}(\mathrm{t}, \tau) \widehat{s u}_{1}^{\dagger}(\mathrm{T}, \tau) \widehat{\mathrm{B}}_{\mathrm{l}+1}(\tau) \mathrm{d} \tau, \\
\widehat{s u}_{\mathrm{l}+1}^{\dagger}(\mathrm{T})=\int_{t_{1}}^{t} \mathrm{I}_{T}(\mathrm{t}, \tau) \widehat{s u}_{1}^{\dagger}(\mathrm{T}, \tau) \widehat{\mathrm{B}}_{\mathrm{l}+1} \mathrm{~d} \tau,
\end{gathered}
$$

where $\gamma_{\text {suT }}=\gamma_{\text {suD }}=\gamma$.

With the introduction of a further variable

$$
\left.X_{5}=\int_{t_{1}}^{t} X_{4} \tau\right) d \tau
$$

the solutions of equation (80) and (81) can be formulated as

$$
\begin{gathered}
X_{1}=\int_{t_{1}}^{t} e^{-\gamma(\mathrm{t}-\tau)} \cos \left(\mathrm{X}_{5}(\mathrm{t})-\mathrm{X}_{5}(\tau)\right) \mathrm{X}_{3}(\tau) \mathrm{d} \tau, \\
\mathrm{X}_{2}=i \int_{t_{1}}^{t} e^{-\gamma(\mathrm{t}-\tau)} \sin \left(\mathrm{X}_{5}(\mathrm{t})-\mathrm{X}_{5}(\tau)\right) \mathrm{X}_{3}(\tau) \mathrm{d} \tau .
\end{gathered}
$$

The correctness of these proposed solutions is proved for example for $\frac{d}{d t} \mathrm{X}_{1}$

where the introduced abbreviations are;

$$
\begin{aligned}
& \mathrm{I}_{\mathrm{D}}=\mathrm{i} \mathrm{g}_{3} e^{-\gamma(\mathrm{t}-\tau)} \cos \left(\mathrm{X}_{5}(\mathrm{t})-\mathrm{X}_{5}(\tau)\right) \\
& \mathrm{I}_{\mathrm{T}}=\mathrm{i} \mathrm{g}_{3} e^{-\gamma(\mathrm{t}-\tau)} \sin \left(\mathrm{X}_{5}(\mathrm{t})-\mathrm{X}_{5}(\tau)\right) .
\end{aligned}
$$

When the damping constant $\gamma$ is sufficient large then $\left|X_{5}(t)-X_{5}(\tau)\right|$ is small and can be approximated by $\alpha|t-\tau|$, where $\alpha$ is a positive constant. Hence, the cosine in (88) can be approximated by $\cos (\alpha|t-\tau|) \approx 1$. Thus, the magnitude of $\widehat{\operatorname{su}}_{1}^{\dagger}(\mathrm{D})$ is approximately given by

$$
\left|\widehat{s u}_{1+1}^{\dagger}(\mathrm{D})\right| \approx\left|\int_{t_{1}}^{t e^{-\gamma(\mathrm{t}-\tau)}} \mathrm{i} \mathrm{g}_{3} \widehat{s u}_{1}^{\dagger}(\mathrm{T}, \tau) \widehat{\mathrm{B}}_{1+1}(\tau) \mathrm{d} \tau\right| \approx \alpha\left|\mathrm{t}-\mathrm{t}_{1}\right| e^{-\gamma \mathrm{t}}
$$

since the approximation $\widehat{s u}_{1}^{\dagger}(\mathrm{T}, \tau) \approx e^{-\gamma \tau}$ is pointed out by $(57)$ and $\widehat{\mathrm{B}}_{1+1}(\tau)$ is assumed to be constant and set to 1 . Hence, $\left|\widehat{s u}_{1}^{\dagger}(\mathrm{D})\right|$ increase a short time with $\mathrm{t}$ until it will be damped by $e^{-\gamma \mathrm{t}}$.

The approximation for $\left|\widehat{s u}_{1+1}^{\dagger}(\mathrm{T})\right|$ is

$$
\left|\widehat{s u}_{1+1}^{\dagger}(\mathrm{T})\right| \approx\left|\int_{t_{0}}^{t} e^{-\gamma(\mathrm{t}-\tau)} \alpha(\mathrm{t}-\tau) \mathrm{ig}_{3} \widehat{\operatorname{su}}_{1}^{\dagger}(\mathrm{T}, \tau) \widehat{\mathrm{B}}_{\mathrm{l}+1}(\tau) \mathrm{d} \tau \approx \alpha\right| \mathrm{t}-\left.\mathrm{t}_{1}\right|^{2}
$$


Hence, $\left|\widehat{s u}_{1+1}^{\dagger}(\mathrm{T})\right|$ increase quadratically with time until the exponential damping dominates.

Now, the next evaluations concern the two signaling proteins $\widehat{\mathrm{pk}}_{\mathrm{l}+1}^{\dagger}$ and $\widehat{\mathrm{ac}}_{\mathrm{l}+1}^{\dagger}$. If the abbreviation $\mathrm{Y}=\mathrm{i} \mathrm{g}_{2}$ $\widehat{s u}_{1+1}^{\dagger}(\mathrm{D}) \quad \widehat{\mathrm{su}}_{1+1}(\mathrm{~T}), Y \in \mathbb{R}$ is introduced, then the corresponding equations of motion are rewritten as

$$
\frac{d}{d t} \widehat{\mathrm{pk}}_{\mathrm{l}+1}^{\dagger}=Y \widehat{\mathrm{ac}}_{\mathrm{l}+1}^{\dagger}, \frac{d}{d t} \widehat{\mathrm{ac}}_{\mathrm{l}+1}^{\dagger}=\mathrm{i} \widehat{\mathrm{pk}}_{l+1}^{\dagger} \text {. }
$$

Due to the previous temporal results for $\left|\widehat{s u}_{1+1}^{\dagger}(\mathrm{D})\right|$ and $\left|\widehat{s u}_{1+1}^{\dagger}(\mathrm{T})\right|$ the approximation $|\mathrm{Y}| \approx \alpha\left|\mathrm{t}-\mathrm{t}_{1}\right|^{3}$ is valid. Consequently, it can be assumed that

$$
\frac{d}{d t} \widehat{\mathrm{pk}}_{1+1}^{\dagger} \approx 0 \text { and } \frac{d}{d t} \widehat{\mathrm{ac}}_{1+1}^{\dagger} \approx 0 .
$$

All in all, it was demonstrated that this approach describes the assembly of a microtubule periodically and stepwise from one location to the next one with D-forms (after hydrolysis of one T-form, (64)), which can be called "hopping" solution or incoherent solution. This was presented by the exemplarily sequential allocation of two D-forms at locations 1 and $1+1$, under the constraints that the initial conditions are: $\widehat{s u}_{1}(\mathrm{D}, \mathrm{t}=0)=\widehat{s u}_{1+1}(\mathrm{D}, \mathrm{t}=0)=0$.

\subsubsection{Shrinkage of the Plus End of Microtubules}

When the polymerization rate (adding rate of GTPs) is lower than the hydrolysis rate of T-forms then a GDP-cap is formed and splayed off. The energy of this "spring apart" is delivered by the release of the hydrolysis energy stored in the microtubule lattice. Cooperatively the free phosphate group is set free. When a T-form hydrolyzes then the free phosphate group and the energy of hydrolysis stored in the microtubule lattice are together released. This energy rapidly peels off the GDP-cap. But, GTP containing subunits still add to the shrinking plus end, if enough are added, then a new GTP cap forms and the microtubule growth resumes ("rescue").

The following, complete local Hamiltonian reverts to equation (64), where protein kinesin $\left(\widehat{\mathrm{pk}}_{1}\right)$ is replaced by kinesin $13\left(\widehat{\mathrm{km}}_{1}\right)$, which enhances the disassembly at the plus end, and the further mediating accessory protein $\widehat{\mathrm{ac}}_{1}$ is replaced by inorganic phosphor $\widehat{\mathrm{ph}}_{1}$. Consequently, the modified Hamiltonian reads

$$
\left.\widehat{\mathrm{H}}_{i n t, 1}=\hbar \mathrm{g}_{2}\left(\widehat{\mathrm{su}}_{1}^{\dagger}(\mathrm{T}) \widehat{\mathrm{su}}_{1}(\mathrm{D}) \widehat{\mathrm{ph}}_{1}^{\dagger} \widehat{\mathrm{kmn}}_{1}+\widehat{\mathrm{knn}}_{1}^{\dagger} \widehat{\mathrm{ph}}_{1} \widehat{s u}_{1}^{\dagger}(\mathrm{D}) \widehat{\mathrm{su}}_{1}(\mathrm{~T})\right)+\hbar \mathrm{g}_{3}\left(\widehat{\mathrm{su}}_{1+1}^{\dagger}(\mathrm{D}) \widehat{s u}_{1}(\mathrm{~T})\right) \widehat{\mathrm{B}}_{1+1}^{\dagger}+\widehat{s u}_{1}^{\dagger}(\mathrm{T}) \widehat{\mathrm{su}}_{\mathrm{l}+1}(\mathrm{D}) \widehat{\mathrm{B}}_{1+1}\right),
$$

In this sub-chapter coherent, running waves solution of the equations of motion are preferred in contradiction to the "hopping solutions" of the preceding sub-chapter. Where, the preconditions are unaltered: $\widehat{\mathrm{B}}_{1}^{\dagger}=\widehat{\mathrm{B}}_{1}=1, \gamma_{\mathrm{ph}}=\gamma_{\mathrm{kin}}=0$, and $\gamma=\gamma_{\text {suT }}=\gamma_{\text {suD }}$, and the fluctuations are neglected. Hence, the modified Heisenberg equations of motion at location 1 read:

$$
\begin{aligned}
& \frac{d}{d t} \widehat{\operatorname{su}}_{1}^{\dagger}(\mathrm{D})=\mathrm{i} \mathrm{g}_{2} \widehat{\mathrm{su}}_{\mathrm{l}}^{\dagger}(\mathrm{T}) \widehat{\mathrm{ph}}_{1}^{\dagger} \widehat{\mathrm{kmn}}_{1}+\mathrm{i} \mathrm{g}_{3} \widehat{\mathrm{su}}_{\mathrm{l}-1}^{\dagger}(\mathrm{T}) \widehat{\mathrm{B}}_{1}-\gamma \widehat{s u}_{1}^{\dagger}(\mathrm{D}), \\
& \frac{d}{d t} \widehat{\operatorname{su}}_{\mathrm{l}}^{\dagger}(\mathrm{T})=\mathrm{i} \mathrm{g}_{2} \widehat{\mathrm{su}}_{\mathrm{l}}^{\dagger}(\mathrm{D}) \widehat{\mathrm{kin}}_{\mathrm{l}}^{\dagger} \widehat{\mathrm{ph}}_{\mathrm{l}}+\mathrm{i} \mathrm{g}_{3} \widehat{\mathrm{su}}_{\mathrm{l}+\mathbf{1}}^{\dagger}(\mathrm{D}) \widehat{\mathrm{B}}_{\mathrm{l}+\mathbf{1}}^{\dagger}-\gamma \widehat{\mathrm{su}}_{\mathrm{l}}^{\dagger}(\mathrm{T}), \\
& \frac{d}{d t} \widehat{\mathrm{kmn}}_{1}^{\dagger}=\mathrm{ig}_{2} \widehat{\mathrm{su}}_{\mathrm{l}}(\mathrm{D}) \widehat{\mathrm{ph}}_{\mathrm{l}}^{\dagger} \widehat{\mathrm{su}}_{1}^{\dagger}(\mathrm{T}), \\
& \frac{d}{d t} \widehat{\mathrm{ph}}_{\mathrm{l}}^{\dagger}=\mathrm{ig} \widehat{\mathrm{su}}_{\mathrm{l}}^{\dagger}(\mathrm{D}) \widehat{\mathrm{kmn}}_{\mathrm{l}}^{\dagger} \widehat{\mathrm{su}}_{\mathrm{l}}(\mathrm{T}) \text {. } \\
& \frac{d}{d t} \widehat{\mathrm{R}}_{\mathrm{T}}^{\dagger}=\mathrm{i} \mathrm{g}_{2} \widehat{\mathrm{R}}_{\mathrm{D}}^{\dagger} \hat{S}_{\mathrm{kin}}^{\dagger} \hat{S}_{\mathrm{ph}}+\mathrm{ig}_{3} \widehat{\mathrm{R}}_{\mathrm{D}}^{\dagger} e^{i \mathrm{~K}}-\gamma \hat{R}_{\mathrm{T}}^{\dagger},(10 \\
& \frac{d}{d t} \hat{S}_{\mathrm{kin}}^{\dagger}=\mathrm{i} \mathrm{g}_{2} \widehat{\mathrm{R}}_{\mathrm{D}} \hat{S}_{\mathrm{ph}}^{\dagger} \widehat{\mathrm{R}}_{\mathrm{T}}^{\dagger} \text {, } \\
& \frac{d}{d t} \hat{S}_{\mathrm{ph}}^{\dagger}=\mathrm{i} \mathrm{g}_{2} \widehat{\mathrm{R}}_{\mathrm{D}}^{\dagger} \hat{S}_{\mathrm{kin}}^{\dagger} \widehat{\mathrm{R}}_{\mathrm{T}} .
\end{aligned}
$$
proposed for the solutions of the equations (95) till (98):

$$
\begin{aligned}
\widehat{\mathrm{su}}_{\mathrm{l}}^{\dagger}(\mathrm{D}) & =\widehat{\mathrm{R}}_{\mathrm{D}}^{\dagger} e^{i \mathrm{Kl} l}, \\
\widehat{\mathrm{su}}_{\mathrm{l}}^{\dagger}(\mathrm{T}) & =\widehat{\mathrm{R}}_{\mathrm{T}}^{\dagger} e^{i \mathrm{~K} \mathrm{l}}, \\
\widehat{\mathrm{kin}}_{\mathrm{l}}^{\dagger} & =\hat{S}_{\mathrm{kin}}^{\dagger} e^{i \mathrm{~K} \mathrm{l}}, \\
\widehat{\mathrm{ph}}_{\mathrm{l}}^{\dagger} & =\hat{S}_{\mathrm{ph}}^{\dagger} e^{i \mathrm{~K} \mathrm{l}} .
\end{aligned}
$$

Insertion of this ansatz into the original differential equations (95) till (98) cast they into the forms:

$$
\frac{d}{d t} \widehat{\mathrm{R}}_{\mathrm{D}}^{\dagger}=\mathrm{i} \mathrm{g}_{2} \hat{R}_{\mathrm{T}}^{\dagger} \hat{S}_{\mathrm{ph}}^{\dagger} \hat{S}_{\mathrm{kin}}+\mathrm{i} \mathrm{g}_{3} \widehat{\mathrm{R}}_{\mathrm{T}}^{\dagger} e^{-i \mathrm{~K}}-\gamma \widehat{\mathrm{R}}_{\mathrm{D}}^{\dagger},(
$$

The renewed replacement of the operators $\widehat{R}_{D}^{\dagger}$ and $\widehat{R}_{T}^{\dagger}$ in the equations (103) and (104) by the expressions

$$
\widehat{\mathrm{R}}_{\mathrm{D}}^{\dagger}=\hat{\rho}_{\mathrm{D}}^{\dagger} e^{-\gamma \mathrm{t}} \text { and } \widehat{\mathrm{R}}_{\mathrm{T}}^{\dagger}=\hat{\rho}_{\mathrm{T}}^{\dagger} e^{-\gamma \mathrm{t}}
$$

reformulate again the differential equations (103) till (104):

$$
\begin{aligned}
& \frac{d}{d t} \hat{\rho}_{\mathrm{D}}^{\dagger}=\mathrm{i}\left(\mathrm{g}_{2} \hat{S}_{\mathrm{ph}}^{\dagger} \hat{S}_{\mathrm{kin}}+\mathrm{g}_{3} e^{-i \mathrm{~K}}\right) \hat{\rho}_{\mathrm{T}}^{\dagger} \equiv \mathrm{i} \mathrm{D} e^{\mathrm{i} \varphi} \hat{\rho}_{\mathrm{T}}^{\dagger},(10) \\
& \frac{d}{d t} \hat{\rho}_{\mathrm{T}}^{\dagger}=\mathrm{i}\left(\mathrm{g}_{2} \hat{S}_{\mathrm{kin}}^{\dagger} \hat{S}_{\mathrm{ph}}+\mathrm{g}_{3} e^{i \mathrm{~K}}\right) \hat{\rho}_{\mathrm{D}}^{\dagger} \equiv \mathrm{i} \mathrm{D} e^{-\mathrm{i} \varphi} \hat{\rho}_{\mathrm{D}}^{\dagger} .
\end{aligned}
$$

The next reformulation cast $\hat{\rho}_{\mathrm{D}}^{\dagger}$ and $\hat{\rho}_{\mathrm{T}}^{\dagger}$ in the forms

$$
\hat{\rho}_{\mathrm{D}}^{\dagger}=e^{i \mathrm{~F}(\mathrm{t})} \rho_{0}, \hat{\rho}_{\mathrm{T}}^{\dagger}=e^{i \mathrm{~F}(\mathrm{t})} e^{-\mathrm{i} \varphi} \rho_{0}
$$


where $F(t)=\int_{t_{0}}^{t} D(\tau) d \tau$ and $\rho_{0} \in \mathbb{R}$.

Consequently, the equations (105) and (106) obtain the modified forms

$$
\begin{gathered}
\frac{d}{d t} \hat{S}_{\mathrm{kin}}^{\dagger}=\mathrm{i} \mathrm{g}_{2} \hat{\rho}_{\mathrm{D}} \hat{\rho}_{\mathrm{T}}^{\dagger} \hat{S}_{\mathrm{ph}}^{\dagger} e^{-2 \gamma \mathrm{t}}=\mathrm{i} \mathrm{g}_{2} \rho_{0}^{2} e^{-2 \gamma \mathrm{t}} e^{-\mathrm{i} \varphi} \hat{S}_{\mathrm{ph}}^{\dagger} \\
\frac{d}{d t} \hat{S}_{\mathrm{ph}}^{\dagger}=\mathrm{ig} \mathrm{g}_{2} \hat{\rho}_{\mathrm{D}}^{\dagger} \hat{\rho}_{\mathrm{T}} \hat{S}_{\mathrm{kin}}^{\dagger} e^{-2 \gamma \mathrm{t}}=i \mathrm{~g}_{2} \rho_{0}{ }^{2} e^{-2 \gamma \mathrm{t}} e^{\mathrm{i} \varphi} \hat{S}_{\mathrm{kin}}^{\dagger}
\end{gathered}
$$

The solutions of these two equations are

$$
\frac{d}{d t} \hat{S}_{\mathrm{ph}}^{\dagger}=\mathrm{i}\left(\frac{d}{d t} \mathrm{H}\right) \mathrm{e}^{\mathrm{i} \mathrm{H}} e^{\mathrm{i} \varphi} \hat{S}_{0}^{\dagger}+\mathrm{e}^{i \mathrm{H}}\left(\frac{d}{d t} \varphi\right) e^{\mathrm{i} \varphi} \hat{S}_{0}^{\dagger}=\mathrm{i} \mathrm{g}_{2} \rho_{0}{ }^{2} e^{-2 \gamma \tau} e^{\mathrm{i} \varphi}\left(\mathrm{e}^{\mathrm{i} \mathrm{H}} \hat{S}_{0}^{\dagger}\right),
$$

because the phase $\varphi$ is assumed to be constant (e.g. $\varphi=$ $\left.\frac{\pi}{2}\right)$, so $\frac{d}{d t} \varphi=0$.

Insertion of the two equations (112) and (113) into expressions (107) and (108) deliver the results:

$$
\begin{aligned}
& \left(\mathrm{g}_{2}\left|\hat{S}_{0}\right|^{2} e^{i \varphi}+\mathrm{g}_{3} e^{-i \mathrm{~K}}\right) \equiv \mathrm{D} e^{i \varphi}, \\
& \left(\mathrm{g}_{2}\left|\hat{S}_{0}\right|^{2} e^{-i \varphi}+\mathrm{g}_{3} e^{i \mathrm{~K}}\right) \equiv \mathrm{D} e^{-i \varphi} .
\end{aligned}
$$

Because D and $\varphi$ are constants the equations (115) and (116) are rewritten

$$
\begin{aligned}
& \left.\left(\mathrm{g}_{2}\left|\hat{S}_{0}\right|-\mathrm{D}\right) e^{i \varphi}+\mathrm{g}_{3} e^{-i \mathrm{~K}}\right)=0, \\
& \left.\left(\mathrm{~g}_{2}\left|\hat{S}_{0}\right|^{2}-\mathrm{D}\right) e^{-i \varphi}+\mathrm{g}_{3} e^{i \mathrm{~K}}\right)=0 .
\end{aligned}
$$

These two equations provide the following solutions:

$$
\mathrm{K}=-\varphi \text { and } \mathrm{D}=\mathrm{g}_{2}\left|\hat{S}_{0}\right|^{2}+\mathrm{g}_{3} .
$$

Thus $F(t)=\int_{t_{0}}^{t} D(\tau) d \tau(109)$ gets simply $F(t)=D t$, if $t_{0}=0$ is stipulated.

Starting from the equations (99) and (100) and retracing all relevant subsequently insertions concerning $\widehat{\mathrm{su}}_{1}^{\dagger}(\mathrm{D})$ and $\widehat{\mathrm{su}}_{1}^{\dagger}(\mathrm{T})$ then the final results represent damped outgoing running waves:

$$
\begin{gathered}
\widehat{\mathrm{su}}_{1}^{\dagger}(\mathrm{D})=e^{i(\mathrm{Dt}-\varphi \mathrm{l})} \rho_{0} e^{-\gamma \mathrm{t}}, \\
\widehat{\mathrm{su}}_{1}^{\dagger}(\mathrm{T})=e^{i(\mathrm{Dt}-\varphi(\mathrm{l}+1))} \rho_{0} e^{-\gamma \mathrm{t}} .
\end{gathered}
$$

The result for $\widehat{\mathrm{su}}_{1}^{\dagger}(\mathrm{D})$ characterizes descriptively that a Dform of the GDT-cap peels-off and gives the plus end the appearance of a frayed end because the wave spreads out along the complete loop of the plus end of a microtubule. The wave solution designed for $\widehat{s u}_{1}^{\dagger}(T)$ only distinguishes by the phase factor $(1+1)$ from the phase factor 1 for $\widehat{\operatorname{su}}_{1}^{\dagger}(\mathrm{D})$, this means a phase difference between both waves.

Notice, the same wave solutions (120) and (121) are also obtained for the assembly of the plus end of a microtubule or the growth of a microtubule in a vertical direction (e.g. GTPcap). Conversely, the previous "hopping solution" described in the previous sub-chapter 4.1. (i) can also be applied in case of the shrinkage of a microtubule. Thus, the two phases of the dynamic instability of a microtubule can be described either by an incoherent hopping solution or by a coherent matter wave solution. However, both types of solutions are obtained under the restriction that fluctuations are neglected.

\section{Conclusions}

The core theme of this contribution is the unifying quantum description of the dynamic self-organization of microtubules. Thus, this article connects the biological processes of microtubules with relevant aspects of the synergetic approach of the self-organization, and with thermodynamic effects. Regarding the quantum dynamical results, a strict differentiation is performed between incoherent and coherent wave solutions (duality of matter waves). However, the coherent wave solutions that correspond to quantum coherence (micro vibrations) are more significant, because they are actually experimentally verified by a bundle of microtubule (EEG waves), Thus, the coherent quantum effects of microtubules can also be enqueued into the set of approved processes of quantum biology.

The main effect of quantum wave solutions is the emergence of new effects like irregular allocation of tubulin subunit into microtubules, the release of GDP-caps at once, the building of spiral waves during the growth of microtubules and their shrinkage. These are effects that distinctly deviate from the classical biologic descriptions. Consequently, the benefit of the knowledge of such effects is twofold. First, they represent an extension of the knowledge of selected biological effects. Second, they might activate to develop medical support for instance for the chemotherapy and opens the way to develop new medicine against cancer. For example, the replacement or complement of the taxol treatment of ovarian and breast cancer cells to retard their rapid cell divisions.

Furthermore, the prosperous combination of the quantum aspect of microtubules with the fundamental impact of kinesin for the intracellular transport open new medical applications for the regulation of physiological processes as higher brain functions (e.g., conscientiousness), tumor suppression, and developmental patterning. Genetic experiments have uncovered important roles of microtubules together with kinesin in the regulation physiological processes. It will be intriguing to explore additional spectacular quantum effects of the microtubule-kinesin system in eukaryotic cells. This expectation is again intertwined with prospective, successful medical applications. 


\section{References}

[1] Alberts B et al. (2002). Molecular Biology of the Cell. Fifth edition, New York, Garland Science.

[2] Brouhard G J, Rice L M (2018). Microtubules dynamics: an interplay of biochemistry and mechanic. Nature Reviews Molecular Cell Biology 19, 451-463.

[3] Lasser M, Tiber J, and Lowery L A (2018). The Role of the Microtubuls.in Neurodevelopmental Disorders. Frontiers in Cellular Neuroscience, 1-36. https//doi.org/10.3389/fncell.201800165.

[4] Latchman, D (1995). Gene Regulation, A Eukaryotic Perspective. Second edition, London, Chapman \& Hall.

[5] Lambert N, Chen Y-N, Cheng Y-C, Li C-M, Chen, G-C, Nori, F (2012). Quantum Biology. Nature Physics/Review 9, 1-9.

[6] Penrose R (1995). Shadows of the Mind: A Search for the Missing Science of Consciousness. Oxford, Oxford University Press.

[7] Cantero M del R, Etchegoyen C V, Perez P L, Scarind L, Centiello F (2018). Bundles of Brain Microtubules Generate Electric Oscillations. www.nature.com/scientificreports, 1-10.

[8] Gutschner M (2020). Discovery of Quantum Vibrations in Microtubules inside brain Neurons Corroborates Controversial 20-Year-Old Theory of Consciousness. https://www.elsevier.com, 1-5.

[9] Kapoor V, Hirst W G, Hentschel Ch, Preibisch St, Reber S (2019). MTrack: Automated Detection, Tracking, and Analysis of Dynamic Microtubules. www.nature.com/scientificreports, $1-12$.

[10] Haken H, Levi P (2012). Synergetic Agents. Weinheim, Germany, Wiley-VCH Verlag.

[11] Levi P (2015). Molecular quantum robotics: particle and wave solutions, illustrated by "leg-over-leg" walking over microtubules. Frontiers in Neurorobotics, Vol. 9, Article 2, 116. doi: 10.3389/fnbot.2015.00002.

[12] Weinberg S (2005). The Quantum Theory of Fields, Vol. I-II Cambridge, Cambridge University Press.

[13] Lodish H, Berk A et al. (2016). Molecular Cell Biology. Fifth edition, New York, Freeman and Company.

[14] Kaneko T, Furuta K Olwa, K, Shintaku H, Kotera H, Yokokawa R (2020). Different motilities of microtubules driven by kinesin 1 and kinesin 14 motors patterned on nanopillars. Science advances, Vol. 6, no. 4., 1-12.

[15] Van Delinder V, Imam Z I, Bachand G (2019). Kinesin motor density and dynamics in gliding microtubules motility. Springer Nature.com, scientific reports, 1-12.

[16] Leng X, Yang Y-M, Zhu R-D, Song C, Weng Y-X, Sui Q (2018). Theoretical Investigations of the Role of Mutations in Dynamics of Kinesin Motor Proteins. J. Phys. Chem B, 122, 17, 453-466.

[17] Hirokawa N, Noda Y, Tanaka Y, Niwa S (2009). Kinesin superfamily motor proteins and intracellular transport. Nature reviews, molecular cell biology, 1-31.

[18] Haken, H (1983). Synergetics, Introduction and Advanced Topics. Berlin, Springer-Verlag.

[19] Haken H (2006). Information and Self-Organization. Third edition. Berlin, Springer-Verlag.

[20] Tzer H T et al. (2020). Topological turbulence in the membrane of a living cell. Letters of Nature Physics, https://doi.org/10.1038/s41567-020-0841-9.

[21] Levi P (2016). A Quantum Field Based approach to describe the Global Molecular Dynamics of Neurotransmitter Cycles. European Journal of Biophysics, Vol. 4, No. 4, 22-41. doi: 10.11648/ej.20180602.12.

[22] Weinberg S (2013). Lectures on Quantum Mechanics. Cambridge, University Press.

[23] Haken H, Wolf H Ch (1994). The Physics of Atoms and Quanta. Fourth edition, Berlin, Springer-Verlag.

[24] Haken H, Wolf H Ch (2004). Molecular Physics and Elements of Quantum Chemistry, Introduction to Experiments and Theory. Second edition, Berlin, Springer-Verlag.

[25] Levi P (2018). Quantum Interactions of Small-Sized Neurotransmitters and of Entangled Ionotropic Receptors Accentuate the Impact of Entanglement to Consciousness. European Journal of Biophysics. Vol. 6, No. 2, 35-52. doi: 10.11648/j.ejb.20180602.12.

[26] Naber G L (1997). Topology, Geometry, and Gauge Fields. Berlin, Springer-Verlag. 